\title{
Distributed Estimation using Bayesian Consensus Filtering
}

\author{
Saptarshi Bandyopadhyay Student Member, IEEE, Soon-Jo Chung Senior Member, IEEE
}

\begin{abstract}
We present the Bayesian consensus filter (BCF) for tracking a moving target using a networked group of sensing agents and achieving consensus on the best estimate of the probability distributions of the target's states. Our BCF framework can incorporate nonlinear target dynamic models, heterogeneous nonlinear measurement models, non-Gaussian uncertainties, and higher-order moments of the locally estimated posterior probability distribution of the target's states obtained using Bayesian filters. If the agents combine their estimated posterior probability distributions using a logarithmic opinion pool, then the sum of Kullback-Leibler divergences between the consensual probability distribution and the local posterior probability distributions is minimized. Rigorous stability and convergence results for the proposed BCF algorithm with single or multiple consensus loops are presented. Communication of probability distributions and computational methods for implementing the $\mathrm{BCF}$ algorithm are discussed along with a numerical example.
\end{abstract}

\section{INTRODUCTION}

In this paper, the term consensus means reaching an agreement across the network, regarding a certain subject of interest called the target dynamics. Distributed and networked groups of agents can sense the target, broadcast the acquired information, and reach an agreement on the gathered information using consensus algorithms. Potential applications of distributed estimation tasks include environment and pollution monitoring, tracking dust or volcanic ash clouds, tracking mobile targets such as flying objects or space debris using distributed sensor networks, etc. Consensus algorithms are extensively studied in controls [1]-[6], distributed optimization [7]-[10], and distributed estimation problems [11] $-[15]$. Strictly speaking, consensus is different from the term distributed estimation, which refers to finding the best estimate of the target, given a distributed network of sensing agents.

Many existing algorithms for distributed estimation [11][20] aim to obtain the estimated mean (first moment of the estimated probability distribution) of the target dynamics across the network, but cannot incorporate nonlinear target dynamics, heterogeneous nonlinear measurement models, non-Gaussian uncertainties, or higher-order moments of the locally estimated posterior probability distribution of the target's states. It is difficult to recursively combine local mean and covariance estimates using a linear consensus algorithm because the dimension of the vector transmitted by each agent increases linearly with time due to correlated process noise [21] and

The authors are with the Department of Aerospace Engineering and Coordinated Science Laboratory, University of Illinois at Urbana-Champaign, Urbana, IL 61801, USA. (email: bandyop2@illinois.edu; sjchung@illinois.edu)

This research was supported by AFOSR grant FA95501210193.

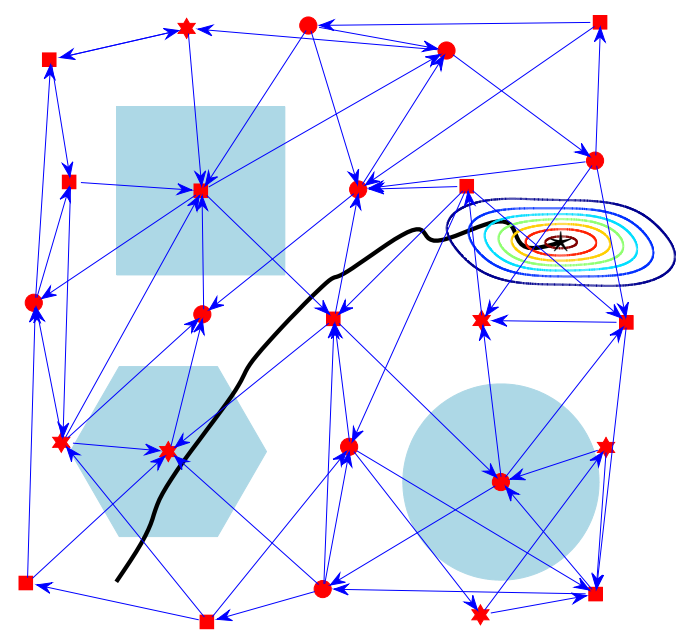

Fig. 1. A heterogeneous sensor network tracks a target, whose current position is marked by $\star$. The sensing region of some sensors are shown in light blue. The sensors use the Hierarchical BCF algorithm to estimate the probability distribution of the target's position and reach a consensus across the network.

the covariance update equation is usually approximated by a consensus gain [22].

Multi-agent tracking or sensing networks are deployed in a distributed fashion when the target dynamics have complex temporal and spatial variations. Hence, it is necessary to preserve the complete information captured in the locally estimated posterior probability distribution of the target's states while achieving consensus across the network. For example, while tracking an orbital debris in space, the uncertainty in position along the direction of velocity is much larger than that orthogonal to the velocity direction, and this extra information is lost if a linear consensus algorithm is used to combine the estimated mean from multiple tracking stations. As shown in Fig 11. the contour plot represents the consensual probability distribution of the target's final position, where the uncertainty along the velocity direction is relatively larger than that in the orthogonal direction.

The main objective of this paper is to extend the scope of distributed estimation algorithms to track targets with general nonlinear dynamic models with stochastic uncertainties, thereby addressing the aforementioned shortcomings. Bayesian filters [23]-[26] recursively calculate the probability density/mass function of the beliefs and update them based on new measurements. The main advantage of Bayesian filters over Kalman filter-based methods [27], [28] for estimation of nonlinear target dynamic models is that no approximation 
is needed during the filtering process. In other words, the complete information about the dynamics and uncertainties of the model can be incorporated in the filtering algorithm. However, Bayesian filtering is computationally expensive. Advances in computational capability have facilitated the implementation of Bayesian filters for robotic localization and mapping [29]-[32] as well as planning and control [33]-[35]. Practical implementation of these algorithms, in their most general form, is achieved using particle filtering [26], [36] and Bayesian programming [37], [38]. This paper focuses on developing a consensus framework for distributed Bayesian filters.

The statistics literature deals with the problem of reaching a consensus among individuals in a complete graph, where each individual's opinion is represented as a probability distribution [39], [40]; and under select conditions, it is shown that consensus is achieved within the group [41]-[43]. Exchange of beliefs in decentralized systems, under communication constraints, is considered in [44], [45]. Algorithms for combining probability distributions within the exponential family, i.e., a limited class of unimodal distributions that can be expressed as an exponential function, are presented in [46], [47]. If the target's states are discrete random variables, then the local estimates can be combined using a tree-search algorithm [48] or a linear consensus algorithm [49]. In contrast, this paper focuses on developing generalized Bayesian consensus algorithms with rigorous convergence analysis for achieving consensus across the network without any assumption on the shape of local prior or posterior probability distributions. The proposed distributed estimation using Bayesian consensus filtering aims to reach an agreement across the network on the best estimate, in the information theoretic sense, of the probability distribution of the target's states.

\section{A. Paper Contributions and Organization}

In this paper, we assume that agents generate their local estimate of the posterior probability distribution of the target's states using Bayesian filters with/without measurement exchange with neighbors. Then, we develop algorithms for combining these local estimates, using the logarithmic opinion pool ( $\log \mathrm{OP})$, to generate the consensual estimate of the probability distribution of the target's states across the network. Finally, we introduce the Bayesian consensus filter (BCF), where the local prior estimates of the target's states are first updated and the local posterior probability distributions are recursively combined during the consensus stage, so that the agents can estimate the consensual probability distribution of the target's states while simultaneously maintaining consensus across the network. The flowchart for the algorithm is shown in Fig. 2 and its pseudo-code is given in Algorithm 1 .

The first contribution of this paper is the LogOP-based consensus algorithm for combining posterior probability distributions during the consensus stage and achieving consensus across the network. As discussed in Section III-B, this is achieved by each agent recursively communicating its posterior probability distribution of the target's states with neighboring agents and updating its estimated probability distribution

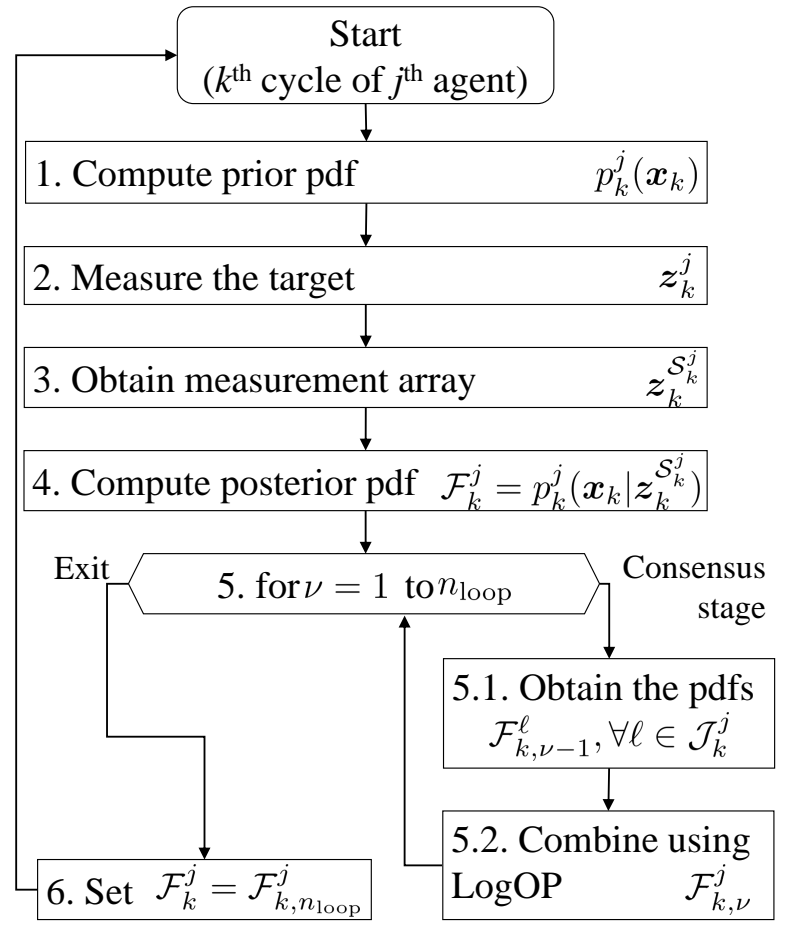

Fig. 2. Flowchart for BCF-LogOP algorithm describing the key steps for a single agent in a single time step. Steps 1-4 represent the Bayesian filtering stage, while step 5 represents the consensus stage.

of the target's states using the LogOP. As shown in Fig. 3 . combining posterior probability distributions using the linear opinion pool (LinOP) typically results in multimodal solutions, which are insensitive to the weights [40]. On the other hand, combining posterior probability distributions using the LogOP typically results in unimodal, less dispersed solutions, thereby indicating a jointly preferred consensual distribution by the network. Moreover, as discussed in Section III-B, the optimal solution does not depend upon the choice of scale of the prior probability distribution and $\log \mathrm{OP}$ is is externally Bayesian [40] (See Fig. 3 (c-d)).

The KL divergence is the measure of the information lost when the consensual estimate is used to approximate the locally estimated posterior probability distributions. In Theorem 6, we show that the LogOP algorithm on a strongly connected (SC) balanced graph minimizes the information lost during the consensus stage, i.e., the consensual probability distribution minimizes the sum of KL divergences with the locally estimated posterior probability distributions. Methods for communicating probability distributions and the effects of inaccuracies on the consensual probability distribution are discussed in Section $\amalg I I-C$

The second contribution of this paper is the BCF algorithm presented in Section IV] As illustrated in Fig. 2 and Algorithm 1 . each agent generates a local estimate of the posterior probability distribution of the target's states using the Bayesian filter. Note that measurement exchanges with neighbors during the Bayesian filtering stage are not mandatory and can be omitted. During the consensus stage, the LogOP algorithm is executed multiple times to reach an agreement across the network. The number of consensus loops $\left(n_{\text {loop }} \in \mathbb{N}\right)$ depends on the second 
largest singular value of the matrix representing a SC balanced communication network topology. Moreover, the convergence conditions for a given number of consensus loops are derived. Note that this consensual probability distribution from the current time step is used as the prior probability distribution in the next time step, as shown in Fig 2. The novel features of the $\mathrm{BCF}$ algorithm are:

- The algorithm can be used to track targets with general nonlinear time-varying target dynamic models.

- The algorithm can be used by a SC balanced network of heterogeneous agents with general nonlinear time-varying measurement models.

- The algorithm achieves global exponential convergence across the network to the consensual probability distribution of the target's states.

- The consensual probability distribution the best estimate, in the information theoretic sense because it minimizes the sum of KL divergences with the locally estimated posterior probability distributions. If a central agent receives all the local posterior probability distributions and is tasked to find the best estimate in the information theoretic sense, then it would also yield the same consensual probability distribution. Hence, we claim to have achieved distributed estimation using the BCF algorithm.

The Hierarchical BCF algorithms, in Section IV-B, is used when some of the agents do not observe the target. In Section $\mathrm{V}$, we apply the Hierarchical BCF algorithm to the problem of tracking orbital debris in space using the space surveillance network on Earth.

\section{B. Notation}

The time index is denoted by a right subscript. For example, $\boldsymbol{x}_{k}$ represents the true states of the target at the $k^{\text {th }}$ time instant. The target is always within the compact state space $\mathcal{X}$, i.e., $\boldsymbol{x}_{k} \in \mathcal{X}, \forall k \in \mathbb{N}$. Also, $\boldsymbol{x}_{1: k}$ represents an array of the true states of the target from the first to the $k^{\text {th }}$ time instant. The agent index is denoted by a lower-case right superscript. For example, $\boldsymbol{z}_{k}^{j}$ represents the measurement taken by the $j^{\text {th }}$ agent at the $k^{\text {th }}$ time instant. The symbol $\mathbb{P}(\cdot)$ refers to probability of an event. $\mathcal{F}_{k}^{j}$ represents the estimated probability density function (pdf) of the target's states over the state space $\mathcal{X}$, by the $j^{\text {th }}$ agent at the $k^{\text {th }}$ time instant. The symbol $p(\cdot)$ also refers to pdf or probability mass function (pmf) over the state space $\mathcal{X}$. During the consensus stage at the $k^{\text {th }}$ time instant, $\mathcal{F}_{k, \nu}^{j}$ represents the local pdf of the target's states by the $j^{\text {th }}$ agent at the $\nu^{\text {th }}$ consensus step and $\mathcal{F}_{k}^{\star}$ represents the consensual pdf to which each $\mathcal{F}_{k, \nu}^{j}$ converges. Let $\mathscr{X}$ be the Borel $\sigma$-algebra on $\mathcal{X}$.

The communication network topology at the $k^{\text {th }}$ time instant is represented by the directed time-varying graph $\mathcal{G}_{k}$, where all the agents of the system form the set of vertices $\mathcal{V}$ (which does not change with time) and the set of directed edges is denoted by $\mathcal{E}_{k}$. The neighbors of the $j^{\text {th }}$ agent at the $k^{\text {th }}$ time instant is the set of agents from which the $j^{\text {th }}$ agent receives information at the $k^{\text {th }}$ time instant and is denoted by $\mathcal{N}_{k}^{j}$, i.e., $\ell \in \mathcal{N}_{k}^{j}$ if and only if $\overrightarrow{\ell j} \in \mathcal{E}_{k}$ for all $\ell, j \in \mathcal{V}$. The set of inclusive neighbors of the $j^{\text {th }}$ agent is denoted by $\mathcal{J}_{k}^{j}:=\mathcal{N}_{k}^{j} \cup\{j\}$.
Let $\mathbb{N}$ and $\mathbb{R}$ be the sets of natural numbers (positive integers) and real numbers respectively. The set of all $m$ by $n$ matrices over the field of real numbers $\mathbb{R}$ is denoted by $\mathbb{R}^{m \times n}$. Let $\lambda$ and $\sigma$ represent the eigenvalue and the singular value of a square matrix. Let $\mathbf{1}=[1,1, \ldots, 1]^{T}, \mathbf{I}, \mathbf{0}$, and $\phi$ be the ones vector, the identity matrix, the zero matrix of appropriate sizes, and the empty set respectively. The symbols $|\cdot|,\lceil\cdot\rceil$, and $\operatorname{sgn}(\cdot)$ represent the absolute value, ceiling function, and signum function respectively. Let $\ln (\cdot)$ and $\log _{c}(\cdot)$ represent the natural logarithm and the logarithm to the base $c$. Finally, $\|\cdot\|_{\ell_{p}}$ represents the $\ell_{p}$ vector norm. The $\mathcal{L}_{p}$ function denotes the set of all functions $f(\boldsymbol{x}): \mathbb{R}^{n_{x}} \rightarrow \mathbb{R}$ with the bounded integral $\left(\int_{\mathcal{X}}|f(\boldsymbol{x})|^{p} d \mu(\boldsymbol{x})\right)^{1 / p}$, where $\mu$ is a measure on $\mathscr{X}$.

\section{PRELIMINARIES}

In this section, we first state four assumptions used throughout this paper and then introduce the problem statement of BCF. Next, we discuss an extension of the Bayesian filter to sensor fusion over a network.

Assumption 1. In this paper, all the algorithms are presented in discrete time.

Assumption 2. The state space $\left(\mathcal{X} \subset \mathbb{R}^{n_{x}}\right)$ is closed and bounded. Hence, by the Heine-Borel theorem (cf. [50, pp. 86]), $\mathcal{X}$ is compact.

Assumption 3. All continuous probability distributions are upper-bounded by some large value $\mathcal{M} \in \mathbb{R}$.

Assumption 4. The inter-agent communication time scale is much faster than the tracking/estimation time scale.

Assumptions 11 and 2 are introduced to discretize the time and bound the state space, so that the algorithms are computationally tractable. Under these assumptions, particle filters [36], approximate grid-based filters, or histogram filters [32] can be used to execute the algorithms developed in this paper. Assumptions 2 and 3 are introduced to take advantage of the results in information theory and measure theory, which deal with bounded functions on compact support. Under Assumption 4, the agents can execute multiple consensus loops within each tracking time step.

We envisage that the results in this paper could be extended to continuous time if the Fokker-Plank equations are solved efficiently [51] and additional issues due to communication delay and time scale separation are addressed. Under Assumption 1. we do not discuss continuous time related issues in this paper. Next, we show that discrete and continuous probability distributions can be handled in a unified manner.

Remark 1. Let $\mathscr{X}$ be the Borel $\sigma$-algebra for $\mathcal{X}$. The probability of a set $\mathscr{A} \in \mathscr{X}$ may be written as the LebesgueStieltjes integral $\mathbb{P}(\mathscr{A})=\int_{\mathscr{A}} p(\boldsymbol{x}) d \mu(\boldsymbol{x})$, where $\mu$ is a measure on $\mathscr{X}$. In the continuous case, $p(\boldsymbol{x})$ is the pdf and $\mu$ is the Lebesgue measure. In the discrete case, $p(\boldsymbol{x})$ is the pmf and $\mu$ is the the counting measure.

Hence, in this paper, we only deal with pdfs over $\mathcal{X}$ with $\mu$ as the Lebesgue measure. Similar arguments will also work for pmfs or mixed probability distributions. 


\section{A. Problem Statement}

Let $\mathcal{X} \subset \mathbb{R}^{n_{x}}$ be the $n_{x}$-dimensional state space of the target. The dynamics of the target in discrete time $\left\{\boldsymbol{x}_{k}, k \in\right.$ $\left.\mathbb{N}, \boldsymbol{x}_{k} \in \mathcal{X}\right\}$ is given by:

$$
\boldsymbol{x}_{k}=\boldsymbol{f}_{k}\left(\boldsymbol{x}_{k-1}, \boldsymbol{v}_{k-1}\right),
$$

where $\boldsymbol{f}_{k}: \mathbb{R}^{n_{x}} \times \mathbb{R}^{n_{v}} \rightarrow \mathbb{R}^{n_{x}}$ is a possibly nonlinear timevarying function of the state $\boldsymbol{x}_{k-1}$ and an independent and identically distributed (i.i.d.) process noise $\boldsymbol{v}_{k-1}$, where $n_{v}$ is the dimension of the process noise vector.

Let $m$ heterogeneous agents simultaneously track this target and estimate the pdf of the target's states (where $m$ does not change with time). The measurement model of the $j^{\text {th }}$ agent is given by:

$$
\boldsymbol{z}_{k}^{j}=\boldsymbol{h}_{k}^{j}\left(\boldsymbol{x}_{k}, \boldsymbol{w}_{k}^{j}\right), \quad \forall j \in\{1, \ldots, m\},
$$

where $\boldsymbol{h}_{k}^{j}: \mathbb{R}^{n_{x}} \times \mathbb{R}^{n_{w j}} \rightarrow \mathbb{R}^{n_{z j}}$ is a possibly nonlinear timevarying function of the state $\boldsymbol{x}_{k}$ and an i.i.d. measurement noise $\boldsymbol{w}_{k}^{j}$, where $n_{z j}, n_{w j}$ are dimensions of the measurement and measurement noise vectors respectively. Note that the measurement model of agents is quite general since it accommodates heterogeneous sensors with various bandwidths, ranges, and noise characteristics and partial state observation.

The objective of the BCF is to estimate the target's states and maintain consensus across the network. This objective is achieved in two steps: (i) each agent locally estimates the pdf of the target's states using a Bayesian filter, and (ii) each agent's local estimate converges to a global estimate during the consensus stage (see Fig. 11). The objective of Bayesian filtering with/without measurement exchange, discussed in Section II-B is to estimate the posterior pdf of the target's states at the $k^{\text {th }}$ time instant, which is denoted by $\mathcal{F}_{k}^{j}, \forall j \in\{1, \ldots, m\}$, using the estimated prior pdf of the target's states $\mathcal{F}_{k-1}^{j}$ from the $(k-1)^{\text {th }}$ time instant and the new measurement array obtained at the $k^{\text {th }}$ time instant. The objective of the consensus stage, discussed in Section III is to guarantee pointwise convergence of each estimated pdf $\mathcal{F}_{k, \nu}^{j}$ to the consensual pdf $\mathcal{F}_{k}^{\star}$.

\section{B. Bayesian Filter with Measurement Exchange}

A Bayesian filter consist of two steps: (i) the prior pdf of the target's states is obtained during the prediction stage, and (ii) the posterior pdf of the target's states is updated using the new measurement array during the update stage [23][26]. The Bayesian filter gives the exact posterior probability distribution, hence it is the best possible estimate of the target from the available information.

Exchange of measurements is optional since heterogeneous agents, with different priors, fields of view, resolutions, tolerances, etc., may not be able to combine measurements from other agents. For example, if a satellite in space and a low flying quadrotor are observing the same target, then they cannot exchange measurements due to their different fields of view. Furthermore, a centralized estimator may not be able to combine measurements from all heterogeneous agents in the network to estimate $p_{k}\left(\boldsymbol{x}_{k} \mid \boldsymbol{z}_{k}^{\{1, \ldots, m\}}\right)$, because it would have to use a common prior for all the agents. Hence, in this paper, we let the individual agents generate their own posterior pdfs of the target's states and then combine them to get the best estimated pdf from the network.

If an agent can combine measurements from another neighboring agent during its update stage, then we call them measurement neighbors. In this section, we extend the Bayesian filter by assuming that each agent transmits its measurements to other agents in the network, and receives the measurements from its measurement neighbors. Let $\boldsymbol{z}_{k}^{\mathcal{S}_{k}^{j}}:=\left\{\boldsymbol{z}_{k}^{\ell}, \forall \ell \in \mathcal{S}_{k}^{j}\right\}$ denote the array of measurements taken at the $k^{\text {th }}$ time instant by the measurement neighbors of the $j^{\text {th }}$ agent, where $\mathcal{S}_{k}^{j} \subseteq \mathcal{J}_{k}^{j}$ denotes the set of measurement neighbors among the inclusive neighbors of the $j^{\text {th }}$ agent. Next, we assume that the prior is available at the initial time.

Assumption 5. For each agent $j$, the initial prior of the states $\mathcal{F}_{0}^{j}=p_{0}^{j}\left(\boldsymbol{x}_{0}\right)$, is assumed to be available. In case no knowledge about $\boldsymbol{x}_{0}$ is available, $\mathcal{F}_{0}^{j}$ is assumed to be uniformly distributed over $\mathcal{X}$.

In Bayesian Filtering with Measurement Exchanges, the $j^{\text {th }}$ agent estimates the posterior pdf of the target's states $\mathcal{F}_{k}^{j}=p_{k}^{j}\left(\boldsymbol{x}_{k} \mid \boldsymbol{z}_{k}^{\mathcal{S}_{k}^{j}}\right)$ at the $k^{\text {th }}$ time instant using the estimated consensual pdf of the target's states $\mathcal{F}_{k-1}^{j}=p_{k-1}^{j}\left(\boldsymbol{x}_{k-1}\right)$ from the $(k-1)^{\text {th }}$ time instant and the new measurement array $\boldsymbol{z}_{k} \mathcal{S}_{k}^{j}$ obtained at the $k^{\text {th }}$ time instant. The prediction stage involves using the target dynamics model (1) to obtain the estimated pdf of the target's states at the $k^{\text {th }}$ time instant via the ChapmanKolmogorov equation:

$$
p_{k}^{j}\left(\boldsymbol{x}_{k}\right)=\int_{\mathcal{X}} p_{k}^{j}\left(\boldsymbol{x}_{k} \mid \boldsymbol{x}_{k-1}\right) p_{k-1}^{j}\left(\boldsymbol{x}_{k-1}\right) d \mu\left(\boldsymbol{x}_{k-1}\right)
$$

The probabilistic model of the state evolution $p_{k}^{j}\left(\boldsymbol{x}_{k} \mid \boldsymbol{x}_{k-1}\right)$ is defined by the target dynamics model (1) and the known statistics of the i.i.d. process noise $\boldsymbol{v}_{k-1}$.

Proposition 1. The new measurement array $\left(\boldsymbol{z}_{k}^{\mathcal{S}_{k}^{j}}\right)$ is used to compute the posterior pdf of the target's states $\left(\mathcal{F}_{k}^{j}=\right.$ $\left.p_{k}^{j}\left(\boldsymbol{x}_{k} \mid \boldsymbol{z}_{k}^{\mathcal{S}_{k}^{j}}\right)\right)$ during the update stage using Bayes' rule (4):

$$
p_{k}^{j}\left(\boldsymbol{x}_{k} \mid \boldsymbol{z}_{k}^{\mathcal{S}_{k}^{j}}\right)=\frac{\left(\prod_{\ell \in \mathcal{S}_{k}^{j}} p_{k}^{\ell}\left(\boldsymbol{z}_{k}^{\ell} \mid \boldsymbol{x}_{k}\right)\right) p_{k}^{j}\left(\boldsymbol{x}_{k}\right)}{\int_{\mathcal{X}}\left(\prod_{\ell \in \mathcal{S}_{k}^{j}} p_{k}^{\ell}\left(\boldsymbol{z}_{k}^{\ell} \mid \boldsymbol{x}_{k}\right)\right) p_{k}^{j}\left(\boldsymbol{x}_{k}\right) d \mu\left(\boldsymbol{x}_{k}\right)},
$$

The likelihood function $p_{k}^{\ell}\left(\boldsymbol{z}_{k}^{\ell} \mid \boldsymbol{x}_{k}\right), \forall \ell \in \mathcal{S}_{k}^{j}$ is defined by the measurement model (2), and the corresponding known statistics of the i.i.d. measurement noise $\boldsymbol{w}_{k}^{\ell}$.

Proof: We need to show that the term $p\left(\boldsymbol{z}_{k}^{\mathcal{S}_{k}^{j}} \mid \boldsymbol{x}_{k}\right)$ in the Bayesian filter [26] simplifies to $\left(\prod_{\ell \in \mathcal{S}_{k}^{j}} p_{k}^{\ell}\left(\boldsymbol{z}_{k}^{\ell} \mid \boldsymbol{x}_{k}\right)\right)$. Let the agents $r, r+1, \ldots$ be measurement neighbors of the $j^{\text {th }}$ agent at the $k^{\text {th }}$ time instant (i.e., $r, r+1, \ldots \in \mathcal{N}_{k}^{j}$ ). Let us define $\boldsymbol{z}_{k}^{\mathcal{S}_{k}^{j} \backslash\{r\}}:=\left\{\boldsymbol{z}_{k}^{\ell}, \forall \ell \in \mathcal{S}_{k}^{j} \backslash\{r\}\right\}$ as the measurement array obtained by the $j^{\text {th }}$ agent at the $k^{\text {th }}$ time instant, which does not contain the measurement from the $r^{\text {th }}$ agent. Since the measurement noise is i.i.d., 2) describes a Markov process of order one, we get: 
$\frac{p\left(\boldsymbol{z}_{k}^{\mathcal{S}_{k}^{j}}, \boldsymbol{x}_{k}\right)}{p\left(\boldsymbol{x}_{k}\right)}=\frac{p\left(\boldsymbol{z}_{k}^{\mathcal{S}_{k}^{j}}, \boldsymbol{x}_{k}\right)}{p\left(\boldsymbol{z}_{k}^{\mathcal{S}_{k}^{j} \backslash\{r\}}, \boldsymbol{x}_{k}\right)} \frac{p\left(\boldsymbol{z}_{k}^{\mathcal{S}_{k}^{j} \backslash\{r\}}, \boldsymbol{x}_{k}\right)}{p\left(\boldsymbol{z}_{k}^{\mathcal{S}_{k}^{j} \backslash\{r, r+1\}}, \boldsymbol{x}_{k}\right)} \ldots \frac{p\left(\boldsymbol{z}_{k}^{j}, \boldsymbol{x}_{k}\right)}{p\left(\boldsymbol{x}_{k}\right)}$ $=p\left(\boldsymbol{z}_{k}^{r} \mid \boldsymbol{z}_{k}^{\mathcal{S}_{k}^{j} \backslash\{r\}}, \boldsymbol{x}_{k}\right) p\left(\boldsymbol{z}_{k}^{(r+1)} \mid \boldsymbol{z}_{k}^{\mathcal{S}_{k}^{j} \backslash\{r, r+1\}}, \boldsymbol{x}_{k}\right) \ldots p\left(\boldsymbol{z}_{k}^{j} \mid \boldsymbol{x}_{k}\right)$

Thus, we obtain $p\left(\boldsymbol{z}_{k}^{\mathcal{S}_{k}^{j}} \mid \boldsymbol{x}_{k}\right)=p\left(\boldsymbol{z}_{k}^{\mathcal{S}_{k}^{j}}, \boldsymbol{x}_{k}\right) / p\left(\boldsymbol{x}_{k}\right)=$ $\prod_{\ell \in \mathcal{S}_{k}^{j}} p_{k}^{\ell}\left(\boldsymbol{z}_{k}^{\ell} \mid \boldsymbol{x}_{k}\right)$.

If the estimates are represented by pmfs, then we can compare these estimates using entropy [52, pp. 13], which is a measure of the uncertainty associated with a random variable or its information content.

Remark 2. Let $\boldsymbol{Y}_{k}^{j}$ be a random variable with the pmf $p_{k}^{j}\left(\boldsymbol{x}_{k} \mid \boldsymbol{z}_{k}^{j}\right)$ given by a stand-alone Bayesian filter [26], $\boldsymbol{Y}_{k}^{\mathcal{S}_{k}^{j}}$ is a random variable with the $\operatorname{pmf} p_{k}^{j}\left(\boldsymbol{x}_{k} \mid \boldsymbol{z}_{k}^{\mathcal{S}_{k}^{j}}\right)$ given by Bayesian filter with measurement exchange, and $H(\cdot)$ refers to the entropy of the random variable. Since $\boldsymbol{Y}_{k}^{\mathcal{S}_{k}^{j}}$ is obtained by conditioning $\boldsymbol{Y}_{k}^{j}$ with $\boldsymbol{z}_{k}^{\mathcal{S}_{k}^{j} \backslash\{j\}}$ because $p_{k}^{j}\left(\boldsymbol{x}_{k} \mid \boldsymbol{z}_{k}^{\mathcal{S}_{k}^{j}}\right)=$ $p_{k}^{j}\left(\boldsymbol{x}_{k} \mid \boldsymbol{z}_{k}^{j}, \boldsymbol{z}_{k}^{\mathcal{S}_{k}^{j} \backslash\{j\}}\right)$, the claim follows from the theorem on conditioning reduces entropy (cf. [52, pp. 27]):

$$
0 \leq I\left(\boldsymbol{Y}_{k}^{j} ; \boldsymbol{z}_{k}^{\mathcal{S}_{k}^{j} \backslash\{j\}}\right)=H\left(\boldsymbol{Y}_{k}^{j}\right)-H\left(\boldsymbol{Y}_{k}^{j} \mid \boldsymbol{z}_{k}^{\mathcal{S}_{k}^{j} \backslash\{j\}}\right),
$$

where $I(\cdot ; \cdot)$ refers to the nonnegative mutual information between two random variables. Since $H\left(\boldsymbol{Y}_{k}^{\mathcal{S}_{k}^{j}}\right)=$ $H\left(\boldsymbol{Y}_{k}^{j} \mid \boldsymbol{z}_{k}^{j} \backslash\{j\}\right) \leq H\left(\boldsymbol{Y}_{k}^{j}\right)$, the estimate given by Bayesian filter with measurement exchange is more accurate than that obtained by a stand-alone Bayesian filter.

Note that (4) is similar to the empirical equation for Independent Likelihood Pool given in [35] and a generalization of the Distributed Sequential Bayesian Estimation Algorithm given in [53]. The structure of (4) ensures that an arbitrary part of the prior distribution does not dominate the measurements. There is no consensus protocol across the network because each agent receives information only from its neighboring agents and never receives measurements (even indirectly) from any other agent in the network.

\section{Combining Probability Distributions}

In this section, we present the algorithms for achieving consensus in probability distributions across the network. As discussed before, the objective of the consensus stage in Algorithm 1 is to guarantee pointwise convergence of each $\mathcal{F}_{k}^{j}$ to a consensual pdf $\mathcal{F}_{k}^{\star}$, which is independent of $j$. This is achieved by each agent recursively transmitting its estimated pdf of the target's states to other agents, receiving estimates of its neighboring agents, and updating its estimate of the target. Let $\mathcal{F}_{k, 0}^{j}=\mathcal{F}_{k}^{j}$ represent the local estimated posterior pdf of the target's states, by the $j^{\text {th }}$ agent at the start of the consensus stage, obtained using Bayesian filters with/without measurement exchange. During each of the $n_{\text {loop }}$ iterations within the consensus stage in Algorithm 11, this estimate is updated as follows:

$$
\mathcal{F}_{k, \nu}^{j}=\mathcal{T}\left(\cup_{\ell \in \mathcal{J}_{k}^{j}}\left\{\mathcal{F}_{k, \nu-1}^{\ell}\right\}\right), \forall j \in\{1, \ldots, m\}, \forall \nu \in \mathbb{N},
$$

where $\mathcal{T}(\cdot)$ is the linear or logarithmic opinion pool for combining the pdf estimates. Note that the problem of measurement neighbors does not arise here since all pdfs are expressed over the complete state space $\mathcal{X}$.

We introduce Lemma 2 to show that pointwise convergence of pdfs is the sufficient condition for convergence of their induced measures in total variation (TV). Let $\mathcal{F}_{1}, \ldots, \lim _{n \rightarrow \infty} \mathcal{F}_{n}, \mathcal{F}^{\star}$ be real-valued measurable functions on $\mathcal{X}, \mathscr{X}$ be the Borel $\sigma$-algebra of $\mathcal{X}$, and $\mathscr{A}$ be any set in $\mathscr{X}$. If $\mu_{\mathcal{F}_{n}}(\mathscr{A})=\int_{\mathscr{A}} \mathcal{F}_{n}(\boldsymbol{x}) d \mu(\boldsymbol{x})$ for any set $\mathscr{A} \in \mathscr{X}$, then $\mu_{\mathcal{F}_{n}}$ is defined as the measure induced by the function $\mathcal{F}_{n}$ on $\mathscr{X}$. Let $\mu_{\mathcal{F}_{n}}, \mu_{\mathcal{F} \star}$ denote the respective induced measures of $\mathcal{F}_{n}, \mathcal{F}^{\star}$ on $\mathscr{X}$.

Definition 3. (Convergence in TV) If $\left\|\mu_{\mathcal{F}_{n}}-\mu_{\mathcal{F}^{\star}}\right\|_{\mathrm{TV}}:=$ $\sup _{\mathscr{A} \in \mathscr{X}}\left|\mu_{\mathcal{F}_{n}}(\mathscr{A})-\mu_{\mathcal{F}^{\star}}(\mathscr{A})\right|$ tends to zero as $n \rightarrow \infty$, then the measure $\mu_{\mathcal{F}_{n}}$ converges to the measure $\mu_{\mathcal{F} \star}$ in TV, i.e., $\lim _{n \rightarrow \infty} \mu_{\mathcal{F}_{n}} \stackrel{\text { T.V. }}{\longrightarrow} \mu_{\mathcal{F}^{\star}}$.

Lemma 2. (Pointwise convergence implies convergence in TV) If $\mathcal{F}_{n}$ converges to $\mathcal{F}^{\star}$ pointwise, i.e., $\lim _{n \rightarrow \infty} \mathcal{F}_{n}=\mathcal{F}^{\star}$ pointwise; then the measure $\mu_{\mathcal{F}_{n}}$ converges in $T V$ to the measure $\mu_{\mathcal{F} \star}$, i.e., $\lim _{n \rightarrow \infty} \mu_{\mathcal{F}_{n}} \stackrel{\stackrel{n}{T . V_{.}} \longrightarrow}{\longrightarrow} \mu_{\mathcal{F} \star}$.

Proof: Similar to the proof of Scheffe's theorem [54, pp. 84], under Assumption 3, using the dominated convergence theorem (cf. [54, Theorem 1.5.6, pp. 23]) for any set $\mathscr{A} \in \mathscr{X}$ gives:

$\lim _{n \rightarrow \infty} \int_{\mathscr{A}} \mathcal{F}_{n}(\boldsymbol{x}) d \mu(\boldsymbol{x})=\int_{\mathscr{A}^{n}} \lim _{n \rightarrow \infty} \mathcal{F}_{n}(\boldsymbol{x}) d \mu(\boldsymbol{x})=\int_{\mathscr{A}} \mathcal{F}^{\star}(\boldsymbol{x}) d \mu(\boldsymbol{x})$.

This relation between measures implies that $\| \lim _{n \rightarrow \infty} \mu_{\mathcal{F}_{n}}-$ $\mu_{\mathcal{F} \star} \|_{\mathrm{TV}}=0$ and $\lim _{n \rightarrow \infty} \mu_{\mathcal{F}_{n}} \stackrel{\text { T.V. }}{\longrightarrow} \mu_{\mathcal{F}^{\star}}$.

\section{A. Consensus using the Linear Opinion Pool}

The first method of combining the estimates is motivated by the linear consensus algorithms widely studied in the literature [5]-[7]. The pdfs are combined using the Linear Opinion Pool (LinOP) of probability measures [39], [40]:

$$
\mathcal{F}_{k, \nu}^{j}=\sum_{\ell \in \mathcal{J}_{k}^{j}} a_{k, \nu-1}^{j \ell} \mathcal{F}_{k, \nu-1}^{\ell}, \forall j \in\{1, \ldots, m\}, \forall \nu \in \mathbb{N},
$$

where $\sum_{\ell \in \mathcal{J}_{k}^{j}} a_{k, \nu-1}^{j \ell}=1$ and the updated pdf $\mathcal{F}_{k, \nu}^{j}$ after the $\nu^{\text {th }}$ consensus loop is a weighted average of the pdfs of the inclusive neighbors $\mathcal{F}_{k, \nu-1}^{\ell}, \forall \ell \in \mathcal{J}_{k}^{j}$ from the $(\nu-1)^{\text {th }}$ consensus loop, at the $k^{\text {th }}$ time instant. Let $\mathcal{W}_{k, \nu}:=$ $\left(\mathcal{F}_{k, \nu}^{1}, \ldots, \mathcal{F}_{k, \nu}^{m}\right)^{T}$ denote an array of pdf estimates of all the agents after the $\nu^{\text {th }}$ consensus loop, then the LinOP $\sqrt{6}$ can be expressed concisely as:

$$
\mathcal{W}_{k, \nu}=P_{k, \nu-1} \mathcal{W}_{k, \nu-1}, \forall \nu \in \mathbb{N}
$$

where $P_{k, \nu-1}$ is a matrix with entries $P_{k, \nu-1}[j, \ell]=a_{k, \nu-1}^{j \ell}$.

Assumption 6. The communication network topology of the multi-agent system $\mathcal{G}_{k}$ is strongly connected (SC). The weights $a_{k, \nu-1}^{j \ell}, \forall j, \ell \in\{1, \ldots, m\}$ and the matrix $P_{k, \nu-1}$ have the following properties: (i) the weights are the same for all consensus loops within each time instant, i.e., $a_{k, \nu-1}^{j \ell}=a_{k}^{j \ell}$ 
and $P_{k, \nu-1}=P_{k}, \forall \nu \in \mathbb{N}$; (ii) the matrix $P_{k}$ conforms with the graph $\mathcal{G}_{k}$, i.e., $a_{k}^{j \ell}>0$ if and only if $\ell \in \mathcal{J}_{k}^{j}$, else $a_{k}^{j \ell}=0$; and (iii) the matrix $P_{k}$ is row stochastic, i.e., $\sum_{\ell=1}^{m} a_{k}^{j \ell}=1$.

Theorem 3. (Consensus using the LinOP on SC Digraphs) Under Assumption 6, using the LinOP (6), each $\mathcal{F}_{k, \nu}^{j}$ asymptotically converges pointwise to the pdf $\mathcal{F}_{k}^{\star}=\sum_{i=1}^{m} \pi_{i} \mathcal{F}_{k, 0}^{i}$ where $\pi=\left[\pi_{1}, \ldots \pi_{m}\right]^{T}$ is the unique stationary distribution of $P_{k}$. Furthermore, their induced measures converge in total variation, i.e., $\lim _{\nu \rightarrow \infty} \mu_{\mathcal{F}_{k, \nu}^{j}} \stackrel{\text { T.V. }}{\longrightarrow} \mu_{\mathcal{F}_{k}^{\star}}, \forall j \in\{1, \ldots, m\}$.

Proof: See Appendix A

Theorem 3 is a generalization of the linear consensus algorithm for combining joint measurement probabilities [55]. Moreover, if $\boldsymbol{\pi}=\mathbf{1}$ and each $\mathcal{F}_{k, 0}^{j}$ is a $\mathcal{L}_{2}$ function, then $\mathcal{F}_{k}^{\star}=\frac{1}{m} \sum_{i=1}^{m} \mathcal{F}_{k, 0}^{i}$ globally minimizes the sum of the squares of $\mathcal{L}_{2}$ distances with the locally estimated posterior pdfs.

As shown in Fig. 3 (a-b), the main difficulty with the LinOP is that the resulting solution is typically multimodal, so no clear choice for jointly preferred estimate emerges from it [40]. Moreover, the LinOP algorithm critically depends on the assumption that the same $0-1$ scale is used by every agent as shown in Fig. 3 (c-d). Hence, better schemes for combining probability distributions are needed for the proposed $\mathrm{BCF}$ algorithm.

\section{B. Consensus using the Logarithmic Opinion Pool}

Note that $\mathcal{F}_{k, \nu}^{j}=p_{k, \nu}^{j}\left(\boldsymbol{x}_{k}\right), \forall \boldsymbol{x}_{k} \in \mathcal{X}$ represents the pdf of the estimated target's states by the $j^{\text {th }}$ agent during the $\nu^{\text {th }}$ consensus loop at the $k^{\text {th }}$ time instant. The LogOP is given as [41]:

$$
\begin{gathered}
\mathcal{F}_{k, \nu}^{j}=p_{k, \nu}^{j}\left(\boldsymbol{x}_{k}\right)=\frac{\prod_{\ell \in \mathcal{J}_{k}^{j}}\left(p_{k, \nu-1}^{\ell}\left(\boldsymbol{x}_{k}\right)\right)^{a_{k, \nu-1}^{j \ell}}}{\int_{\mathcal{X}} \prod_{\ell \in \mathcal{J}_{k}^{j}}\left(p_{k, \nu-1}^{\ell}\left(\boldsymbol{x}_{k}\right)\right)^{a_{k, \nu-1}^{j \ell}} d \mu\left(\boldsymbol{x}_{k}\right)}, \\
\forall j \in\{1, \ldots, m\}, \forall \nu \in \mathbb{N},
\end{gathered}
$$

where $\sum_{\ell \in \mathcal{J}_{k, \nu-1}^{j}} a_{k, \nu-1}^{j \ell}=1$ and the integral in the denominator of $(8)$ is finite. Thus the updated pdf $\mathcal{F}_{k, \nu}^{j}$ after the $\nu^{\text {th }}$ consensus loop is the weighted geometric average of the pdfs of the inclusive neighbors $\mathcal{F}_{k, \nu-1}^{\ell}, \forall \ell \in \mathcal{J}_{k}^{j}$ from the $(\nu-1)^{\text {th }}$ consensus loop, at the $k^{\text {th }}$ time instant. As shown in Fig. 3 (a-b), the LogOP solution is typically unimodal and less dispersed, indicating a consensual estimate jointly preferred by the network [40]. As shown in Fig. 3 (c-d), the LogOP solution is invariant under under rescaling of individual degrees of belief, hence it preserves an important credo of uni-Bayesian decision theory; i.e., the optimal decision should not depend upon the choice of scale for the utility function or prior probability distribution [56]. When the parameter space is finite and a $0-1$ probability scale is adopted, the LogOP is equivalent to the Nash product [57]. Note that if the local probability distribution of the target's states is inherently multimodal, as shown in Fig. 3 (e-f), then LogOP preserves this multimodal nature while combining these local estimates. The most compelling reason for using $\operatorname{LogOP}$ is that it is

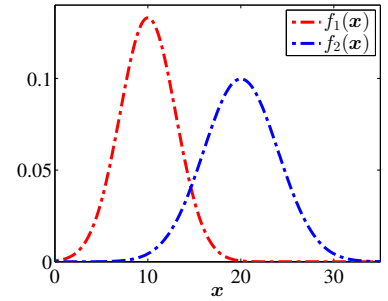

(a)

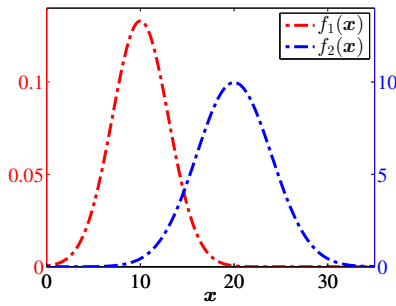

(c)

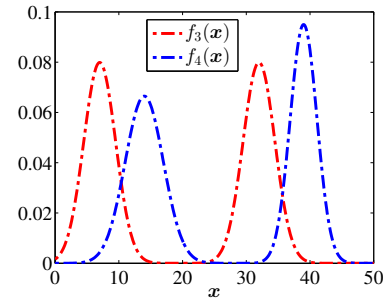

(e)

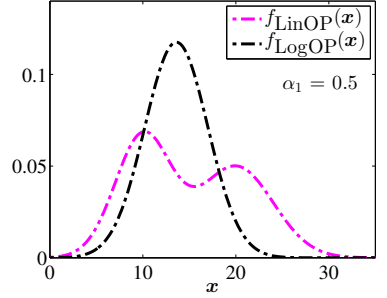

(b)

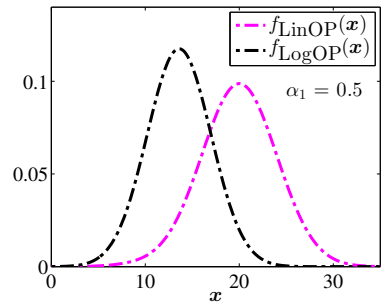

(d)

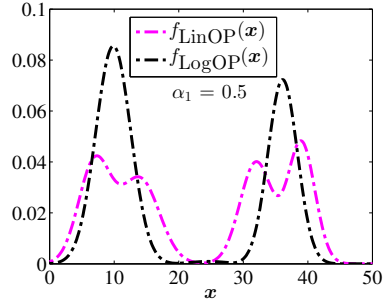

(f)
Fig. 3. In (a), two unimodal pdfs $f_{1}(\boldsymbol{x})$ and $f_{2}(\boldsymbol{x})$ are shown. In (b), these pdfs are combined using the LinOP and LogOP using the weight $\alpha_{1}=0.5$, i.e., $f_{\mathrm{LinOP}}(\boldsymbol{x})=\left(\alpha_{1} f_{1}(\boldsymbol{x})+\left(1-\alpha_{1}\right) f_{2}(\boldsymbol{x})\right)$ and $f_{\mathrm{LogOP}}(\boldsymbol{x})=$ $\left(f_{1}^{\alpha_{1}} \times f_{2}^{\left(1-\alpha_{1}\right)}\right) /\left(\int_{\mathcal{X}}\left(f_{1}^{\alpha_{1}} \times f_{2}^{\left(1-\alpha_{1}\right)}\right) d \mu(\boldsymbol{x})\right)$. Note that the LinOP solution is multimodal while the LogOP solution is unimodal, indicating a consensual pdf.

In (c), the scale of the function $f_{2}(\boldsymbol{x})$ is changed to $0-100$ from the standard $0-1$ scale. In (d), the normalized LinOP solution changes drastically but the LogOP solution remains unaffected.

In (e), the pdfs $f_{3}(\boldsymbol{x})$ and $f_{4}(\boldsymbol{x})$ have bimodal nature. In (f), the LogOP solution preserves this bimodal nature.

externally Bayesian; i.e., finding the consensus distribution commutes with the process of revising distributions using a commonly agreed likelihood distribution. Thus [40]:

$$
\begin{gathered}
\mathcal{T}\left(\cup_{\ell \in \mathcal{J}_{k}^{j}}\left\{\frac{l(\boldsymbol{x}) p_{k}^{\ell}(\boldsymbol{x})}{\int_{\mathcal{X}} l(\boldsymbol{x}) p_{k}^{\ell}(\boldsymbol{x}) d \mu(\boldsymbol{x})}\right\}\right) \\
=\frac{l(\boldsymbol{x}) \mathcal{T}\left(\cup_{\ell \in \mathcal{J}_{k}^{j}}\left\{p_{k}^{\ell}(\boldsymbol{x})\right\}\right)}{\int_{\mathcal{X}} l(\boldsymbol{x}) \mathcal{T}\left(\cup_{\ell \in \mathcal{J}_{k}^{j}}\left\{p_{k}^{\ell}(\boldsymbol{x})\right\}\right) d \mu(\boldsymbol{x})},
\end{gathered}
$$

where $\mathcal{T}(\cdot)$ refers to the $\operatorname{LogOP}(8), p_{k}^{\ell}(\boldsymbol{x}), \forall \ell \in \mathcal{J}_{k}^{j}$ are pdfs on $\mathcal{X}$ and $l(\boldsymbol{x})$ is an arbitrary likelihood pdf on $\mathcal{X}$. Due to these advantages, $\operatorname{LogOP}$ is used for combining prior distributions [58] and conditional random fields for natural language processing tasks [59]. Next, we present consensus theorems using the LogOP.

Assumption 7. The local estimated pdf at the start of the consensus stage is positive everywhere, i.e., $\mathcal{F}_{k, 0}^{j}=p_{k, 0}^{j}\left(\boldsymbol{x}_{k}\right)>$ $0, \forall \boldsymbol{x}_{k} \in \mathcal{X}, \forall j \in\{1, \ldots, m\}$.

Assumption 7 is introduced to avoid regions with zero probability, since they would constitute vetoes and unduly great emphasis would get placed on them. Moreover, the LogOP 
guarantees that $\mathcal{F}_{k, \nu}^{j}$ will remain positive for all subsequent consensus loop.

Definition 4. ( $\mathcal{H}_{k, \nu}^{j}$ vector for $\log O P$ ) For the purpose of analysis, let us choose $\boldsymbol{\psi}_{k} \in \mathcal{X}$ such that $p_{k, \nu}^{j}\left(\boldsymbol{\psi}_{k}\right)>0, \forall j \in$ $\{1, \ldots, m\}, \forall \nu \in \mathbb{N}$. Let us define $\mathcal{H}_{k, \nu}^{j}:=\ln \left[\frac{p_{k, \nu}^{j}\left(\boldsymbol{x}_{k}\right)}{p_{k, \nu}^{j}\left(\boldsymbol{\psi}_{k}\right)}\right]$. Under Assumption $7 \mathcal{H}_{k, \nu}^{j}$ is a well-defined function, but need not be a $\mathcal{L}_{1}$ function. Then, by simple algebraic manipulation of [8], we get [60]:

$$
\begin{gathered}
\frac{p_{k, \nu}^{j}\left(\boldsymbol{x}_{k}\right)}{p_{k, \nu}^{j}\left(\boldsymbol{\psi}_{k}\right)}=\frac{\left(\frac{\prod_{\ell \in \mathcal{J}_{k}^{j}}\left(p_{k, \nu-1}^{\ell}\left(\boldsymbol{x}_{k}\right)\right)^{a_{k, \nu-1}^{j \ell}}}{\int_{\mathcal{X}} \prod_{\ell \in \mathcal{J}_{k}^{j}}\left(p_{k, \nu-1}^{\ell}\left(\boldsymbol{x}_{k}\right)\right)^{a_{k, \nu-1}^{j \ell}} d \mu\left(\boldsymbol{x}_{k}\right)}\right)}{\left(\frac{\prod_{\ell \in \mathcal{J}_{k}^{j}}\left(p_{k, \nu-1}^{\ell}\left(\boldsymbol{\psi}_{k}\right)\right)^{a_{k, \nu-1}^{j \ell}}}{\int_{\mathcal{X}} \prod_{\ell \in \mathcal{J}_{k}^{j}}\left(p_{k, \nu-1}^{\ell}\left(\boldsymbol{x}_{k}\right)\right)^{a_{k, \nu-1}^{j \ell} d \mu\left(\boldsymbol{x}_{k}\right)}}\right)}, \\
\mathcal{H}_{k, \nu}^{j}=\sum_{\ell \in \mathcal{J}_{k}^{j}} a_{k, \nu-1}^{j \ell} \mathcal{H}_{k, \nu-1}^{\ell}, \forall j \in\{1, \ldots, m\}, \nu \in \mathbb{N} .
\end{gathered}
$$

Note that (10) is similar to the LinOP (6). Let $\mathcal{U}_{k, \nu}:=$ $\left(\mathcal{H}_{k, \nu}^{1}, \ldots, \mathcal{H}_{k, \nu}^{m}\right)^{T}$ be an array of the estimates of all the agents during the $\nu^{\text {th }}$ consensus loop at the $k^{\text {th }}$ time instant, then the equation (10) can be expressed concisely as:

$$
\mathcal{U}_{k, \nu}=P_{k, \nu-1} \mathcal{U}_{k, \nu-1}, \forall \nu \in \mathbb{N},
$$

where $P_{k, \nu-1}$ is a matrix with entries $a_{k, \nu-1}^{j l}$.

Thus we are able to use the highly nonlinear LogOP for combining the pdf estimates, but we have reduced the complexity of the problem to that of consensus using the LinOP.

Theorem 4. (Consensus using the LogOP on SC Digraphs) Under Assumptions 6 and 77 using the $\log O P$ (8), each $\mathcal{F}_{k, \nu}^{j}$ asymptotically converges pointwise to the pdf $\mathcal{F}_{k}$ given by:

$$
\mathcal{F}_{k}^{\star}=p_{k}^{\star}\left(\boldsymbol{x}_{k}\right)=\frac{\prod_{i=1}^{m}\left(p_{k, 0}^{i}\left(\boldsymbol{x}_{k}\right)\right)^{\pi_{i}}}{\int_{\mathcal{X}} \prod_{i=1}^{m}\left(p_{k, 0}^{i}\left(\boldsymbol{x}_{k}\right)\right)^{\pi_{i}} d \mu\left(\boldsymbol{x}_{k}\right)},
$$

where $\pi$ is the unique stationary distribution of $P_{k}$. Furthermore, their induced measures converge in total variation, i.e., $\lim _{\nu \rightarrow \infty} \mu_{\mathcal{F}_{k, \nu}^{j}} \stackrel{T . V .}{\longrightarrow} \mu_{\mathcal{F}_{k}^{\star}}, \forall j \in\{1, \ldots, m\}$.

Proof: Similar to the proof of Theorem 3 each $\mathcal{H}_{k, \nu}^{j}$ converges pointwise to $\mathcal{H}_{k}^{\star}=\boldsymbol{\pi}^{T} \mathcal{U}_{k, 0}=\sum_{i=1}^{m} \pi_{i} \mathcal{H}_{k, 0}^{i}$ asymptotically. We additionally need to show that convergence of $\mathcal{H}_{k, \nu}^{j}$ to $\mathcal{H}_{k}^{\star}$ implies pointwise convergence of $\mathcal{F}_{k, \nu}^{j}$ to $\mathcal{F}_{k}^{\star}$. We have $\forall \boldsymbol{x}_{k} \in \mathcal{X}$ :

$$
\lim _{\nu \rightarrow \infty}\left(\ln p_{k, \nu}^{j}\left(\boldsymbol{x}_{k}\right)-\ln p_{k, \nu}^{j}\left(\boldsymbol{\psi}_{k}\right)\right)=\ln p_{k}^{\star}\left(\boldsymbol{x}_{k}\right)-\ln p_{k}^{\star}\left(\boldsymbol{\psi}_{k}\right) .
$$

We claim $\exists \overline{\boldsymbol{\psi}}_{k} \in \mathcal{X}$ such that $\lim _{\nu \rightarrow \infty} p_{k, \nu}^{j}\left(\overline{\boldsymbol{\psi}}_{k}\right)=$ $p_{k}^{\star}\left(\overline{\boldsymbol{\psi}}_{k}\right)$. If this claim is untrue, then $0<$ $\lim _{\nu \rightarrow \infty} p_{k, \nu}^{j}\left(\boldsymbol{x}_{k}\right)<p_{k}^{\star}\left(\boldsymbol{x}_{k}\right), \forall \boldsymbol{x}_{k} \in \mathcal{X}$ or vice versa. Hence $\int_{\mathcal{X}} \lim _{\nu \rightarrow \infty} p_{k, \nu}^{j}\left(\boldsymbol{x}_{k}\right) d \mu\left(\boldsymbol{x}_{k}\right)=1<\int_{\mathcal{X}} p_{k}^{\star}\left(\boldsymbol{x}_{k}\right) d \mu\left(\boldsymbol{x}_{k}\right)$, which results in contradiction since $p_{k}^{\star}\left(\boldsymbol{x}_{k}\right)$ is also a pdf. Hence, substituting $\overline{\boldsymbol{\psi}}_{k}$ into equation 13 gives $\lim _{\nu \rightarrow \infty} p_{k, \nu}^{j}\left(\boldsymbol{x}_{k}\right)=p_{k}^{\star}\left(\boldsymbol{x}_{k}\right), \forall \boldsymbol{x}_{k} \in \mathcal{X}$. Thus each $\mathcal{F}_{k, \nu}^{j}$ converges pointwise to the consensual pdf $\mathcal{F}_{k}^{\star}$ given by (12). By Lemma 2, the measure induced by $\mathcal{F}_{k, \nu}^{j}$ on $\mathscr{X}$ converges in total variation to the measure induced by $\mathcal{F}_{k}^{\star}$ on $\mathscr{X}$, i.e., $\lim _{\nu \rightarrow \infty} \mu_{\mathcal{F}_{k, \nu}^{j}} \stackrel{\text { T.V. }}{\longrightarrow} \mu_{\mathcal{F}_{k}^{*}}$.

Since Perron-Frobenius theorem only yields asymptotic convergence, we next discuss the algorithm for achieving global exponential convergence using balanced graphs.

Assumption 8. In addition to Assumption 6 the weights $a_{k}^{j \ell}$ are such that the digraph $\mathcal{G}_{k}$ is balanced. Hence for every vertex, the in-degree equals the out-degree, i.e., $\sum_{\ell \in \mathcal{J}_{k}^{j}} a_{k}^{j \ell}=$ $\sum_{r \mid j \in \mathcal{J}_{k}^{r}} a_{k}^{r j}$, where $j, \ell, r \in\{1, \ldots, m\}$.

Theorem 5. (Consensus using the LogOP on SC Balanced Digraphs) Under Assumption 7 and 8 using the LogOP (8), each $\mathcal{F}_{k, \nu}^{j}$ globally exponentially converges pointwise to the pdf $\mathcal{F}_{k}^{\star}$ given by:

$$
\mathcal{F}_{k}^{\star}=p_{k}^{\star}\left(\boldsymbol{x}_{k}\right)=\frac{\prod_{i=1}^{m}\left(p_{k, 0}^{i}\left(\boldsymbol{x}_{k}\right)\right)^{\frac{1}{m}}}{\int_{\mathcal{X}} \prod_{i=1}^{m}\left(p_{k, 0}^{i}\left(\boldsymbol{x}_{k}\right)\right)^{\frac{1}{m}} d \mu\left(\boldsymbol{x}_{k}\right)}
$$

at a rate faster or equal to $\sqrt{\lambda_{m-1}\left(P_{k}^{T} P_{k}\right)}=\sigma_{m-1}\left(P_{k}\right)$. Furthermore, their induced measures globally exponentially converge in total variation, i.e., $\lim _{\nu \rightarrow \infty} \mu_{\mathcal{F}_{k, \nu}^{j}} \stackrel{\text { T.V. }}{\longrightarrow} \mu_{\mathcal{F}_{k}^{\star}}, \forall j \in$ $\{1, \ldots, m\}$.

Proof: Since Assumption 8 is stronger than Assumption 6. we get $\lim _{\nu \rightarrow \infty} P_{k}^{\nu}=\mathbf{1} \boldsymbol{\pi}^{T}$. Moreover, since $P_{k}$ is also a column stochastic matrix, therefore $\pi=\frac{1}{m} \mathbf{1}$ is its left eigenvector corresponding to the eigenvalue 1 , i.e., $P_{k}^{T} \frac{1}{m} \mathbf{1}=$ $1 \frac{1}{m} \mathbf{1}$ and satisfying the normalizing condition. Hence, we get $\lim _{\nu \rightarrow \infty} P_{k}^{\nu}=\frac{1}{m} \mathbf{1 1}^{T}$ and each $\mathcal{H}_{k, \nu}^{j}$ converges pointwise to $\mathcal{H}_{k}^{\star}=\frac{1}{m} \mathbf{1}^{T} \mathcal{U}_{k, 0}=\frac{1}{m} \sum_{i=1}^{m} \mathcal{H}_{k, 0}^{i}$.

From the proof of Theorem 4, we get that each $\mathcal{F}_{k, \nu}^{j}$ converges pointwise to the consensual pdf $\mathcal{F}_{k}^{\star}$ given by (14). Note that $\mathcal{F}_{k, \nu}^{j}$ are $\mathcal{L}_{1}$ functions but $\mathcal{H}_{k, \nu}^{j}$ need not be $\mathcal{L}_{1}$ functions. Let $V_{\mathrm{tr}}=\left[\frac{1}{\sqrt{m}} \mathbf{1}, V_{\mathrm{s}}\right]$ be the orthonormal matrix of eigenvectors of the symmetric primitive matrix $P_{k}^{T} P_{k}$. By spectral decomposition [61], we get:

$$
V_{\mathrm{tr}}^{T} P_{k}^{T} P_{k} V_{\mathrm{tr}}=\left[\begin{array}{cc}
1 & \mathbf{0}^{1 \times(m-1)} \\
\mathbf{0}^{(m-1) \times 1} & V_{\mathrm{s}}^{T} P_{k}^{T} P_{k} V_{\mathrm{s}}
\end{array}\right],
$$

where $\frac{1}{m} \mathbf{1}^{T} P_{k}^{T} P_{k} \mathbf{1}=1, \frac{1}{\sqrt{m}} \mathbf{1}^{T} P_{k}^{T} P_{k} V_{\mathrm{s}}=\mathbf{0}^{1 \times(m-1)}$, and $V_{\mathrm{s}}^{T} P_{k}^{T} P_{k} \mathbf{1} \frac{1}{\sqrt{m}}=\mathbf{0}^{(m-1) \times 1}$ are used. Since the eigenvectors are orthonormal, $V_{s} V_{s}^{T}+\frac{1}{m} \mathbf{1 1}^{T}=\mathbf{I}$. The rate at which $\mathcal{U}_{k, \nu}$ synchronizes to $\frac{1}{\sqrt{m}} \mathbf{1}$ (or $\mathcal{U}_{k}^{\star}$ ) is equal to the rate at which $V_{\mathrm{s}}^{T} \mathcal{U}_{k, \nu} \rightarrow \mathbf{0}^{(m-1) \times 1}$. Pre-multiplying 111 by $V_{s}^{T}$ and substituting $V_{s}^{T} \mathbf{1}=0$ results in:

$$
\begin{aligned}
V_{\mathrm{s}}^{T} \mathcal{U}_{k, \nu} & =V_{\mathrm{s}}^{T} P_{k}\left(V_{s} V_{s}^{T}+\frac{1}{m} \mathbf{1 1}^{T}\right) \mathcal{U}_{k, \nu-1} \\
& =V_{\mathrm{s}}^{T} P_{k} V_{s} V_{s}^{T} \mathcal{U}_{k, \nu-1} .
\end{aligned}
$$

Let $\mathbf{z}_{k, \nu}=V_{\mathrm{s}}^{T} \mathcal{U}_{k, \nu}$. The corresponding virtual dynamics is represented by $\mathbf{z}_{k, \nu}=\left(V_{\mathrm{s}}^{T} P_{k} V_{s}\right) \mathbf{z}_{k, \nu-1}$, which has both $V_{\mathrm{s}}^{T} \mathcal{U}_{k, \nu}$ and $\mathbf{0}$ as particular solutions. Let $\Phi_{k, \nu}=\mathbf{z}_{k, \nu}^{T} \mathbf{z}_{k, \nu}$ be a candidate Lyapunov function for this dynamics. Expanding this gives: 
$\Phi_{k, \nu}=\mathbf{z}_{k, \nu-1}^{T} V_{\mathrm{s}}^{T} P_{k}^{T} P_{k} V_{s} \mathbf{z}_{k, \nu-1} \leq\left(\lambda_{\max }\left(V_{\mathrm{s}}^{T} P_{k}^{T} P_{k} V_{s}\right)\right) \Phi_{k, \nu-1}$.

Note that $V_{\mathrm{s}}^{T} P_{k}^{T} P_{k} V_{s}$ contains all the eigenvalues of $P_{k}^{T} P_{k}$ other than 1 . Hence $\lambda_{\max }\left(V_{\mathrm{s}}^{T} P_{k}^{T} P_{k} V_{s}\right)=\lambda_{m-1}\left(P_{k}^{T} P_{k}\right)<1$ and $\Phi_{k, \nu}$ globally exponentially vanishes with a rate faster or equal to $\lambda_{m-1}\left(P_{k}^{T} P_{k}\right)$. Hence each $\mathcal{H}_{k, \nu}^{j}$ globally exponentially converges pointwise to $\mathcal{H}_{k}^{\star}$ with a rate faster or equal to $\sqrt{\lambda_{m-1}\left(P_{k}^{T} P_{k}\right)}=\sigma_{m-1}\left(P_{k}\right)$.

Next, we need to find the rate of convergence of $\mathcal{F}_{k, \nu}^{j}$ to $\mathcal{F}_{k}^{\star}$. From the exponential convergence of $\mathcal{H}_{k, \nu}^{j}$, we get:

$$
\begin{aligned}
& \left|\ln \left[\frac{p_{k, \nu}^{j}\left(\boldsymbol{x}_{k}\right)}{p_{k}^{\star}\left(\boldsymbol{x}_{k}\right)} \frac{p_{k}^{\star}\left(\boldsymbol{\psi}_{k}\right)}{p_{k, \nu}^{j}\left(\boldsymbol{\psi}_{k}\right)}\right]\right| \\
& \leq \sigma_{m-1}\left(P_{k}\right)\left|\ln \left[\frac{p_{k, \nu-1}^{j}\left(\boldsymbol{x}_{k}\right)}{p_{k}^{\star}\left(\boldsymbol{x}_{k}\right)} \frac{p_{k}^{\star}\left(\boldsymbol{\psi}_{k}\right)}{p_{k, \nu-1}^{j}\left(\boldsymbol{\psi}_{k}\right)}\right]\right| .
\end{aligned}
$$

Let us define the function $\alpha_{k, \nu}^{j}\left(\boldsymbol{x}_{k}\right)$ such that $\alpha_{k, \nu}^{j}\left(\boldsymbol{x}_{k}\right)=$ $\left[\frac{p_{k, \nu}^{j}\left(\boldsymbol{x}_{k}\right)}{p_{k}^{\star}\left(\boldsymbol{x}_{k}\right)} \frac{p_{k}^{\star}\left(\boldsymbol{\psi}_{k}\right)}{p_{k, \nu}^{j}\left(\boldsymbol{\psi}_{k}\right)}\right]$ if $p_{k, \nu}^{j}\left(\boldsymbol{x}_{k}\right) p_{k}^{\star}\left(\boldsymbol{\psi}_{k}\right) \geq p_{k}^{\star}\left(\boldsymbol{x}_{k}\right) p_{k, \nu}^{j}\left(\boldsymbol{\psi}_{k}\right)$ and $\alpha_{k, \nu}^{j}\left(\boldsymbol{x}_{k}\right)=\left[\frac{p_{k}^{\star}\left(\boldsymbol{x}_{k}\right)}{p_{k, \nu}^{j}\left(\boldsymbol{x}_{k}\right)} \frac{p_{k, \nu}^{j}\left(\boldsymbol{\psi}_{k}\right)}{p_{k}^{\star}\left(\boldsymbol{\psi}_{k}\right)}\right]$ otherwise. Note that $\alpha_{k, \nu}^{j}\left(\boldsymbol{x}_{k}\right)$ is a continuous function since it is a product of continuous functions. Since $\alpha_{k, \nu}^{j}\left(\boldsymbol{x}_{k}\right) \geq 1$ and $\ln \left(\alpha_{k, \nu}^{j}\left(\boldsymbol{x}_{k}\right)\right) \geq 0, \forall \boldsymbol{x}_{k} \in$ $\mathcal{X}$, (15) simplifies to:

$$
\begin{aligned}
\ln \left(\alpha_{k, \nu}^{j}\left(\boldsymbol{x}_{k}\right)\right) & \leq \sigma_{m-1}\left(P_{k}\right) \ln \left(\alpha_{k, \nu-1}^{j}\left(\boldsymbol{x}_{k}\right)\right) . \\
\alpha_{k, \nu}^{j}\left(\boldsymbol{x}_{k}\right) & \leq\left(\alpha_{k, 0}^{j}\left(\boldsymbol{x}_{k}\right)\right)^{\left(\sigma_{m-1}\left(P_{k}\right)\right)^{\nu}} .
\end{aligned}
$$

Since $p_{k, \nu}^{j}\left(\boldsymbol{x}_{k}\right)$ tends to $p_{k}^{\star}\left(\boldsymbol{x}_{k}\right)$, i.e., $\lim _{\nu \rightarrow \infty} \alpha_{k, \nu}^{j}\left(\boldsymbol{x}_{k}\right)=1$, we can write (16) as:

$$
\alpha_{k, \nu}^{j}\left(\boldsymbol{x}_{k}\right)-1 \leq\left(\alpha_{k, 0}^{j}\left(\boldsymbol{x}_{k}\right)\right)^{\left(\sigma_{m-1}\left(P_{k}\right)\right)^{\nu}}-1^{\left(\sigma_{m-1}\left(P_{k}\right)\right)^{\nu}}
$$

Using the mean value theorem (cf. [50]), the right hand side of 17 can be simplified to 18 , for some $c \in\left[1, \alpha_{k, 0}^{j}\left(\boldsymbol{x}_{k}\right)\right]$.

$$
\begin{aligned}
& \left(\alpha_{k, 0}^{j}\left(\boldsymbol{x}_{k}\right)\right)^{\left(\sigma_{m-1}\left(P_{k}\right)\right)^{\nu}}-1^{\left(\sigma_{m-1}\left(P_{k}\right)\right)^{\nu}} \\
& =\left(\sigma_{m-1}\left(P_{k}\right)\right)^{\nu}\left(c^{\left(\sigma_{m-1}\left(P_{k}\right)\right)^{\nu}-1}\right)\left(\alpha_{k, 0}^{j}\left(\boldsymbol{x}_{k}\right)-1\right) .
\end{aligned}
$$

As $\sigma_{m-1}\left(P_{k}\right)<1$, the maximum value of $\left(c^{\left(\sigma_{m-1}\left(P_{k}\right)\right)^{\nu}-1}\right)$ is 1 . Substituting this result into 17 gives:

$$
\alpha_{k, \nu}^{j}\left(\boldsymbol{x}_{k}\right)-1 \leq\left(\sigma_{m-1}\left(P_{k}\right)\right)^{\nu}\left(\alpha_{k, 0}^{j}\left(\boldsymbol{x}_{k}\right)-1\right) .
$$

Hence $\alpha_{k, \nu}^{j}\left(\boldsymbol{x}_{k}\right)$ exponentially converges to 1 with a rate faster or equal to $\sigma_{m-1}\left(P_{k}\right)$. Irrespective of the orientation of $\alpha_{k, \nu}^{j}\left(\boldsymbol{x}_{k}\right)$ and $\alpha_{k, 0}^{j}\left(\boldsymbol{x}_{k}\right), 19$ can be written as 20 by multiplying with $\frac{1}{\alpha_{k, \nu}^{j}\left(\boldsymbol{x}_{k}\right)}$ or $\frac{1}{\alpha_{k, 0}^{j}\left(\boldsymbol{x}_{k}\right)}$, and then with $p_{k}^{\star}\left(\boldsymbol{x}_{k}\right)$.

$$
\begin{aligned}
& \left|\frac{p_{k}^{\star}\left(\boldsymbol{\psi}_{k}\right)}{p_{k, \nu}^{j}\left(\boldsymbol{\psi}_{k}\right)} p_{k, \nu}^{j}\left(\boldsymbol{x}_{k}\right)-p_{k}^{\star}\left(\boldsymbol{x}_{k}\right)\right| \\
& \leq\left(\sigma_{m-1}\left(P_{k}\right)\right)^{\nu}\left|\frac{p_{k}^{\star}\left(\boldsymbol{\psi}_{k}\right)}{p_{k, 0}^{j}\left(\boldsymbol{\psi}_{k}\right)} p_{k, 0}^{j}\left(\boldsymbol{x}_{k}\right)-p_{k}^{\star}\left(\boldsymbol{x}_{k}\right)\right| .
\end{aligned}
$$

As shown in the proof of Theorem 4 , we can choose $\tilde{\psi}_{k} \in \mathcal{X}$ such that $p_{k, 0}^{j}\left(\tilde{\boldsymbol{\psi}}_{k}\right)=p_{k}^{\star}\left(\tilde{\boldsymbol{\psi}}_{k}\right)$. Now we discuss two cases to reduce the left hand side of 20 to $\left|p_{k, \nu}^{j}\left(\boldsymbol{x}_{k}\right)-p_{k}^{\star}\left(\boldsymbol{x}_{k}\right)\right|$.

$$
\begin{aligned}
& \left|\frac{p_{k}^{\star}\left(\tilde{\boldsymbol{\psi}}_{k}\right)}{p_{k, \nu}^{j}\left(\tilde{\boldsymbol{\psi}}_{k}\right)} p_{k, \nu}^{j}\left(\boldsymbol{x}_{k}\right)-p_{k}^{\star}\left(\boldsymbol{x}_{k}\right)\right| \\
& \int\left|p_{k, \nu}^{j}\left(\boldsymbol{x}_{k}\right)-p_{k}^{\star}\left(\boldsymbol{x}_{k}\right)+\left(\frac{p_{k}^{\star}\left(\tilde{\boldsymbol{\psi}}_{k}\right)}{p_{k, \nu}^{j}\left(\tilde{\boldsymbol{\psi}}_{k}\right)}-1\right) p_{k, \nu}^{j}\left(\boldsymbol{x}_{k}\right)\right| \\
& =\left\{\begin{array}{c}
\text { if } \frac{p_{k}^{\star}\left(\tilde{\boldsymbol{\psi}}_{k}\right)}{p_{k, \nu}^{j}\left(\tilde{\boldsymbol{\psi}}_{k}\right)} \geq 1 \\
\left|p_{k}^{\star}\left(\boldsymbol{x}_{k}\right)-p_{k, \nu}^{j}\left(\boldsymbol{x}_{k}\right)+\left(1-\frac{p_{k}^{\star}\left(\tilde{\boldsymbol{\psi}}_{k}\right)}{p_{k, \nu}^{j}\left(\tilde{\boldsymbol{\psi}}_{k}\right)}\right) p_{k, \nu}^{j}\left(\boldsymbol{x}_{k}\right)\right|
\end{array}\right. \\
& \text { if } \frac{p_{k}^{\star}\left(\tilde{\boldsymbol{\psi}}_{k}\right)}{p_{k, \nu}^{j}\left(\tilde{\boldsymbol{\psi}}_{k}\right)}<1 \\
& \geq\left|p_{k}^{\star}\left(\boldsymbol{x}_{k}\right)-p_{k, \nu}^{j}\left(\boldsymbol{x}_{k}\right)\right| \text {. }
\end{aligned}
$$

Hence, for both the cases, we are able to simplify $(20)$ to:

$$
\left|p_{k, \nu}^{j}\left(\boldsymbol{x}_{k}\right)-p_{k}^{\star}\left(\boldsymbol{x}_{k}\right)\right| \leq\left(\sigma_{m-1}\left(P_{k}\right)\right)^{\nu}\left|p_{k, 0}^{j}\left(\boldsymbol{x}_{k}\right)-p_{k}^{\star}\left(\boldsymbol{x}_{k}\right)\right| .
$$

Thus each $\mathcal{F}_{k, \nu}^{j}=p_{k, \nu}^{j}\left(\boldsymbol{x}_{k}\right)$ globally exponentially converges to $\mathcal{F}_{k}^{\star}=p_{k}^{\star}\left(\boldsymbol{x}_{k}\right)$ with a rate faster or equal to $\sigma_{m-1}\left(P_{k}\right)$.

The KL divergence is a measure of the information lost when the consensual pdf is used to approximate the locally estimated posterior pdfs. We now show that the consensual pdf $\mathcal{F}_{k}^{\star}$ obtained using Theorem 5, which is the weighted geometric average of the locally estimated posterior pdfs $\mathcal{F}_{k, 0}^{j}, \forall j \in\{1, \ldots, m\}$, minimizes the information lost during the consensus stage because it minimizes the sum of KL divergences with those pdfs.

Theorem 6. The consensual pdf $\mathcal{F}_{k}^{\star}$ given by (14) globally minimizes the sum of Kullback-Leibler $(K L)$ divergences with the locally estimated posterior pdfs at the start of the consensus stage $\mathcal{F}_{k, 0}^{j}, \forall j \in\{1, \ldots, m\}$, i.e.,

$$
\mathcal{F}_{k}^{\star}=\arg \min _{\rho \in \mathscr{L}_{1}(\mathcal{X})} \sum_{i=1}^{m} D_{K L}\left(\rho \| \mathcal{F}_{k, 0}^{i}\right),
$$

where $\mathscr{L}_{1}(\mathcal{X})$ is the set of all pdfs over the state space $\mathcal{X}$ satisfying Assumption 7 .

Proof: The sum of the KL divergences of a pdf $\rho \in$ $\mathscr{L}_{1}(\mathcal{X})$ with the locally estimated posterior pdfs is given by:

$$
\begin{aligned}
& \sum_{i=1}^{m} D_{K L}\left(\rho \| \mathcal{F}_{k, 0}^{i}\right)= \\
& \sum_{i=1}^{m} \int_{\mathcal{X}}\left(\rho\left(\boldsymbol{x}_{k}\right) \ln \left(\rho\left(\boldsymbol{x}_{k}\right)\right)-\rho\left(\boldsymbol{x}_{k}\right) \ln \left(p_{k, 0}^{i}\left(\boldsymbol{x}_{k}\right)\right)\right) d \mu\left(\boldsymbol{x}_{k}\right) .
\end{aligned}
$$

Under Assumption 7., $D_{K L}\left(\rho \| \mathcal{F}_{k, 0}^{i}\right)$ is well defined for all agents. Differentiating (22) with respect to $\rho$ using Leibniz integral rule [54, Theorem A.5.1, pp. 372], and equating it to zero gives:

$$
\sum_{i=1}^{m} \int_{\mathcal{X}}\left(\ln \left(\rho\left(\boldsymbol{x}_{k}\right)\right)+1-\ln \left(p_{k, 0}^{i}\left(\boldsymbol{x}_{k}\right)\right)\right) d \mu\left(\boldsymbol{x}_{k}\right)=0,
$$

where $\rho^{\star}\left(\boldsymbol{x}_{k}\right)=\frac{1}{e} \prod_{i=1}^{m}\left(p_{k, 0}^{i}\left(\boldsymbol{x}_{k}\right)\right)^{1 / m}$ is the solution to 23. The projection of $\rho^{\star}$ on the set $\mathscr{L}_{1}(\mathcal{X})$, obtained by 
normalizing $\rho^{\star}$ to 1 , is the consensual pdf $\mathcal{F}_{k}^{\star} \in \mathscr{L}_{1}(\mathcal{X})$ given by (14).

The KL divergence is a convex function of pdf pairs [52, Theorem 2.7.2, pp. 30], hence the sum of KL divergences 22 is a convex function of $\rho$. If $\rho_{1}, \rho_{2}, \ldots, \rho_{n} \in \mathscr{L}_{1}(\mathcal{X})$ and $\eta_{1}, \eta_{2}, \ldots, \eta_{n} \in[0,1]$ such that $\sum_{i=1}^{n} \eta_{i}=1$, then $\rho^{\dagger}=\sum_{i=1}^{n} \eta_{i} \rho_{i} \in \mathscr{L}_{1}(\mathcal{X})$; because (i) since $\rho_{i}\left(\boldsymbol{x}_{k}\right)>$ $0, \forall \boldsymbol{x}_{k} \in \mathcal{X}, \forall i \in\{1, \ldots, n\}$ therefore $\rho^{\dagger}\left(\boldsymbol{x}_{k}\right)>0, \forall \boldsymbol{x}_{k} \in \mathcal{X}$; and (ii) since $\int_{\mathcal{X}} \rho_{i}\left(\boldsymbol{x}_{k}\right) d \mu\left(\boldsymbol{x}_{k}\right)=1, \forall i \in\{1, \ldots, n\}$ therefore $\int_{\mathcal{X}} \rho^{\dagger}\left(\boldsymbol{x}_{k}\right) d \mu\left(\boldsymbol{x}_{k}\right)=1$. Moreover, since $\mathcal{X}$ is a compact set, therefore $\mathscr{L}_{1}(\mathcal{X})$ is a closed set. Hence $\mathscr{L}_{1}(\mathcal{X})$ is a closed convex set. Hence (21) is a convex optimization problem.

The gradient of $\sum_{i=1}^{m} D_{K L}\left(\rho \| \mathcal{F}_{k, 0}^{i}\right)$ evaluated at $\mathcal{F}_{k}^{\star}$ is a constant, i.e.,

$$
\left.\frac{d}{d \rho} \sum_{i=1}^{m} D_{K L}\left(\rho \| \mathcal{F}_{k, 0}^{i}\right)\right|_{\rho=\mathcal{F}_{k}^{\star}}=m \ln \frac{e}{\int_{\mathcal{X}} \prod_{i=1}^{m}\left(p_{k, 0}^{i}\left(\boldsymbol{x}_{k}\right)\right)^{\frac{1}{m}} d \mu\left(\boldsymbol{x}_{k}\right)}
$$

This indicates that for further minimizing the convex cost function, we have to change the normalizing constant of $\mathcal{F}_{k}^{\star}$, which will result in exiting the set $\mathscr{L}_{1}(\mathcal{X})$. Hence $\mathcal{F}_{k}^{\star}$ is the global minimum of the convex cost function 21 in the convex set $\mathscr{L}_{1}(\mathcal{X})$. This is illustrated using a simple example in Fig. 4.

Another proof approach involves taking the logarithm, in the KL divergence formula, to the base $c:=\left(\int_{\mathcal{X}} \prod_{i=1}^{m}\left(p_{k, 0}^{i}\left(\boldsymbol{x}_{k}\right)\right)^{\frac{\mathcal{Y}}{m}} d \mu\left(\boldsymbol{x}_{k}\right)\right)$. Then differentiating $\sum_{i=1}^{m} D_{K L}\left(\rho \| \mathcal{F}_{k, 0}^{i}\right)$ with respect to $\rho$ gives:

$$
\sum_{i=1}^{m} \int_{\mathcal{X}}\left(\log _{c}\left(\rho\left(\boldsymbol{x}_{k}\right)\right)+1-\log _{c}\left(p_{k, 0}^{i}\left(\boldsymbol{x}_{k}\right)\right)\right) d \mu\left(\boldsymbol{x}_{k}\right)=0,
$$

which is minimized by $\mathcal{F}_{k}^{\star}$. Hence $\mathcal{F}_{k}^{\star}$ is indeed the global minimum of the convex optimization problem (21).

Note that if a central agent receives all the locally estimated posterior pdfs $\left(\mathcal{F}_{k, 0}^{j}, \forall j \in\{1, \ldots, m\}\right)$ and is tasked to find the best estimate in the information theoretic sense, then it would also yield the same consensual pdf $\mathcal{F}_{k}^{\star}$ given by (14). Hence we claim to have achieved distributed estimation using this algorithm.

In Remark 5, we state that the methods for recursively combining probability distributions to reach a consensual distribution are limited to $\mathrm{LinOP}, \log \mathrm{OP}$, and their affine combinations.

Remark 5. The LinOP and LogOP methods for combining probability distributions can be generalized by the g-QuasiLinear Opinion Pool (g-QLOP), which is described by the following equation:

$$
\begin{aligned}
\mathcal{F}_{k, \nu}^{j}= & \frac{g^{-1}\left(\sum_{\ell \in \mathcal{J}_{k}^{j}} \alpha_{k, \nu-1}^{j \ell} g\left(\mathcal{F}_{k, \nu-1}^{\ell}\right)\right)}{\int_{\mathcal{X}} g^{-1}\left(\sum_{\ell \in \mathcal{J}_{k}^{j}} \alpha_{k, \nu-1}^{j \ell} g\left(\mathcal{F}_{k, \nu-1}^{\ell}\right)\right) d \mu\left(\boldsymbol{x}_{k}\right)}, \\
& \forall j \in\{1, \ldots, m\}, \forall \nu \in \mathbb{N},
\end{aligned}
$$

where $g$ is a continuous, strictly monotone function. It is shown in [60] that, other than the linear combination of LinOP and $\operatorname{LogOP}$, there is no function $g$ for which the final consensus can be expressed by the following equation:

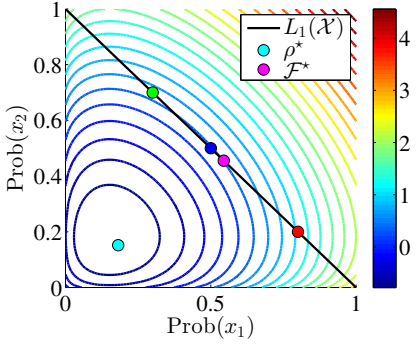

(a)

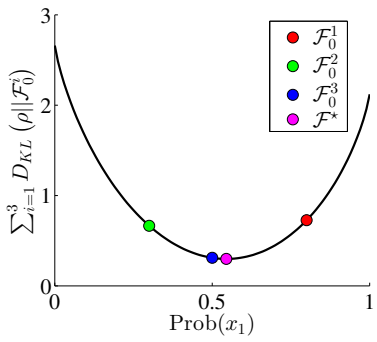

(b)
Fig. 4. Let the discrete state space $\mathcal{X}$ have only two states $x_{1}$ and $x_{2}$. All valid pmfs must lie on the set $\mathscr{L}_{1}(\mathcal{X})$ where $\mathbb{P}\left(x_{1}\right)+\mathbb{P}\left(x_{2}\right)=1$. Given three initial pmfs $\mathcal{F}_{0}^{i}, i=\{1,2,3\}$, the objective is to find the pmf that globally minimizes the convex cost function $\sum_{i=1}^{3} D_{K L}\left(\rho \| \mathcal{F}_{0}^{i}\right)$. In (a), $\rho^{\star}=\frac{1}{e} \prod_{i=1}^{3}\left(\mathcal{F}_{0}^{i}\right)^{1 / m}$ globally minimizes the cost function, but it does not lie on $\mathscr{L}_{1}(\mathcal{X})$. In (b), $\mathcal{F}^{\star} \in \mathscr{L}_{1}(\mathcal{X})$, which is the projection of $\rho^{\star}$ on the set $\mathscr{L}_{1}(\mathcal{X})$ obtained by normalizing $\rho^{\star}$ to 1 , indeed globally minimizes the cost function on the set $\mathscr{L}_{1}(\mathcal{X})$.

$$
\begin{aligned}
\lim _{\nu \rightarrow \infty} \mathcal{F}_{k, \nu}^{j}= & \mathcal{F}_{k}^{\star}=\frac{g^{-1}\left(\sum_{j=1}^{m} \pi_{j} g\left(\mathcal{F}_{k, 0}^{\ell}\right)\right)}{\int_{\mathcal{X}} g^{-1}\left(\sum_{j=1}^{m} \pi_{j} g\left(\mathcal{F}_{k, 0}^{\ell}\right)\right) d \mu\left(\boldsymbol{x}_{k}\right)}, \\
& \forall j \in\{1, \ldots, m\}, \forall \nu \in \mathbb{N},
\end{aligned}
$$

where $\pi$ is the unique stationary solution. Moreover, the function $g$ is said to be k-Markovian if the scheme for combining probability distribution (24) yields the consensus 25) for every regular communication network topology and for all initial positive densities. It is also shown that $g$ is k-Markovian if and only if the g-QLOP is either LinOP or $\log \mathrm{OP}$ [60].

\section{Communicating Probability Distributions}

The consensus algorithms using either LinOP or LogOP need the estimated pdfs to be communicated to other agents in the network. We propose to adopt the following methods for communicating pdfs.

The first approach involves approximating the pdf by a weighted sum of Gaussians and then transmitting this approximate distribution. Let $\mathcal{N}\left(\boldsymbol{x}_{k}-m_{i}, B_{i}\right)$ denote the Gaussian density function, where the mean is the $n_{x}$-vector $m_{i}$ and the covariance is the positive-definite symmetric matrix $B_{i}$. The Gaussian sum approximations lemma of [62, pp. 213] states that any pdf $\mathcal{F}=p\left(\boldsymbol{x}_{k}\right)$ can be approximated as closely as desired in the $\mathscr{L}_{1}\left(\mathbb{R}^{n_{x}}\right)$ space by a pdf of the form $\hat{\mathcal{F}}=\hat{p}\left(\boldsymbol{x}_{k}\right)=\sum_{i=1}^{n_{g}} \alpha_{i} \mathcal{N}\left(\boldsymbol{x}_{k}-m_{i}, B_{i}\right)$, for some integer $n_{g}$ and positive scalars $\alpha_{i}$ with $\sum_{i=1}^{n_{g}} \alpha_{i}=1$. For an acceptable communication error $\varepsilon_{\text {comm }}>0$, there exists $n_{g}, \alpha_{i}, m_{i}$ and $B_{i}$ such that $\|\mathcal{F}-\hat{\mathcal{F}}\|_{\mathcal{L}_{1}} \leq \varepsilon_{\text {comm }}$. Several techniques for estimating the parameters are discussed in the Gaussian mixture model literature, like maximum likelihood (ML) and maximum a posteriori (MAP) parameter estimation [63]- [65]. Hence, in order to communicate the pdf $\hat{\mathcal{F}}$, the agent needs to transmit $\frac{1}{2} n_{g} n_{x}\left(n_{x}+3\right)$ real numbers.

Let us study the effect of this communication error $\varepsilon_{\text {comm }}$ on the LinOP consensual distribution. Let $\tilde{\mathcal{F}}_{k, \nu}^{j}$ be the LinOP solution after combining local pdfs corrupted by communication error, i.e., $\tilde{\mathcal{F}}_{k, \nu}^{j}:=\mathcal{T}\left(\cup_{\ell \in \mathcal{J}_{k}^{j}}\left\{\hat{\mathcal{F}}_{k, \nu-1}^{\ell}\right\}\right)$ where $\mathcal{T}(\cdot)$ is 
LinOP 6. We prove by induction that $\left\|\mathcal{F}_{k, \nu}^{j}-\tilde{\mathcal{F}}_{k, \nu}^{j}\right\|_{\mathcal{L}_{1}} \leq$ $\nu \varepsilon_{\text {comm }}, \forall \nu \in \mathbb{N}$, where $\mathcal{F}_{k, \nu}^{j}$ is the true solution obtained from uncorrupted local pdfs. As the basis of induction holds, the inductive step for the $\nu^{\text {th }}$ consensus step is as follows:

$$
\begin{aligned}
& \left\|\mathcal{F}_{k, \nu}^{j}-\tilde{\mathcal{F}}_{k, \nu}^{j}\right\|_{\mathcal{L}_{1}} \leq \sum_{\ell \in \mathcal{J}_{k}^{j}} a_{k, \nu-1}^{j \ell}\left(\left\|\mathcal{F}_{k, \nu-1}^{\ell}-\tilde{\mathcal{F}}_{k, \nu-1}^{\ell}\right\|_{\mathcal{L}_{1}}\right. \\
& \left.\quad+\left\|\tilde{\mathcal{F}}_{k, \nu-1}^{\ell}-\hat{\mathcal{F}}_{k, \nu-1}^{\ell}\right\|_{\mathcal{L}_{1}}\right) \leq(\nu-1) \varepsilon_{\text {comm }}+\varepsilon_{\text {comm }} .
\end{aligned}
$$

Similarly, it follows from the proof of Theorem 5 that the LogOP solution after $n_{\text {loop }}$ iterations $\left(\tilde{\mathcal{F}}_{k, n_{\text {loop }}}^{j}\right)$, under communication inaccuracies, is always within a ball of $n_{\text {loop }} \varepsilon_{\text {comm }}$ radius from the true solution using $\operatorname{LogOP}\left(\mathcal{F}_{k, n_{\text {loop }}}^{j}\right)$ in the $\mathscr{L}_{1}(\mathcal{X})$ space, i.e., $\left\|\mathcal{F}_{k, n_{\text {loop }}}^{j}-\tilde{\mathcal{F}}_{k, n_{\text {loop }}}^{j}\right\|_{\mathcal{L}_{1}} \leq n_{\text {loop }} \varepsilon_{\text {comm }}$.

If particle filters are used to evaluate the Bayesian filter and combine the pdfs [36], [65], then the resampled particles represent the agent's estimated pdf of the target. Hence communicating pdfs is equivalent to transmitting these resampled particles.

The information theoretic approach for communicating pdfs is discussed in [66]. Let the local pdf $\mathcal{F}_{k, \nu}^{j}$ be transmitted over a communication channel using a finite sequence and the pdf $\hat{\mathcal{F}}_{k, \nu}^{j}$ is reconstructed by the other agent. For a given error threshold, the minimum rate such that the variational distortion between $\mathcal{F}_{k, \nu}^{j}$ and $\hat{\mathcal{F}}_{k, \nu}^{j}$ is bounded by the error threshold, is given by the mutual information between transmitted and received finite sequences.

Now that we have established that communication of pdfs is possible, let us discuss the complete BCF algorithm.

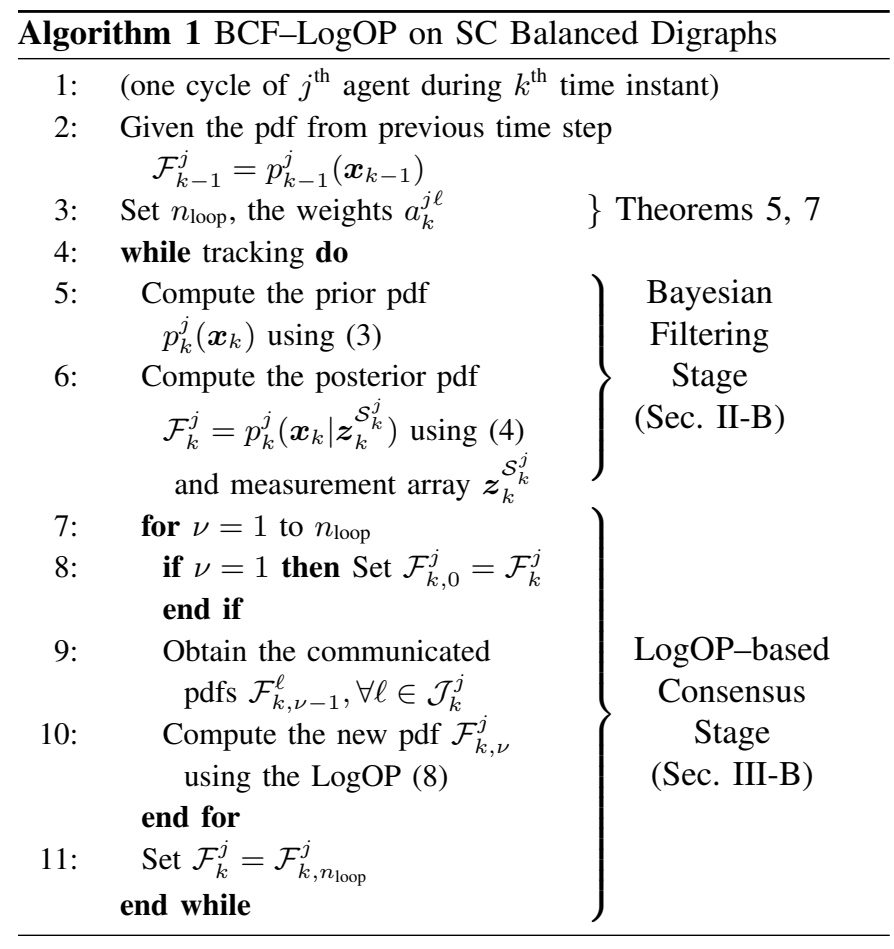

\section{Main Algorithms: BAYEsian Consensus FILTERING}

In this section, we finally solve the complete problem statement for BCF discussed in Section II-A and Algorithm 1 .
We also introduce an hierarchical algorithm that can be used when some agents in the network fail to observe the target.

\section{A. Bayesian Consensus Filtering}

The BCF is performed in two steps: (i) each agent locally estimates the pdf of the target's states using a Bayesian filter with/without measurements from neighboring agents, as discussed in Section II-B and (ii) during the consensus stage, each agent recursively transmits its pdf estimate of the target's states to other agents, receives estimates of its neighboring agents, and combines them using the LogOP as discussed in Section III-B According to [67], this strategy of first updating the local estimate and then combining these local estimates to achieve a consensus is stable and gives the best performance in comparison with other update-combine strategies. In this section, we compute the number of consensus loops ( $n_{\text {loop }}$ in Algorithm 1) needed to reach a satisfactory consensus estimate across the network and discuss the convergence of this algorithm.

Definition 6. (Disagreement vector $\boldsymbol{\theta}_{k, \nu}$ ) Let us define $\boldsymbol{\theta}_{k, \nu}:=\left(\theta_{k, \nu}^{1}, \ldots, \theta_{k, \nu}^{m}\right)^{T}$, where $\theta_{k, \nu}^{j}:=\left\|\mathcal{F}_{k, \nu}^{j}-\mathcal{F}_{k}^{\star}\right\|_{\mathcal{L}_{1}}$. Since the $\mathcal{L}_{1}$ distance between pdfs is upper bounded by 2 , the $\ell_{2}$ norm of the disagreement vector $\left(\left\|\boldsymbol{\theta}_{k, \nu}\right\|_{\ell_{2}}\right)$ is upper bounded by $2 \sqrt{m}$.

This conservative bound is used to obtain the minimum number of consensus loops for achieving $\varepsilon$-consensus across the network, while tracking a moving target. Let us now quantify the divergence of the local pdfs during the Bayesian filtering stage.

Definition 7. (Error propagation dynamics $\boldsymbol{\Gamma}(\cdot)$ ) Let us assume that the dynamics of the $\ell_{2}$ norm of the disagreement vector during the Bayesian filtering stage can be obtained from the target dynamics and measurement models (1) and (2). The error propagation dynamics $\boldsymbol{\Gamma}(\cdot)$ estimates the maximum divergence of the local pdfs during the Bayesian filtering stage, i.e., $\left\|\boldsymbol{\theta}_{k, 0}\right\|_{\ell_{2}} \leq \boldsymbol{\Gamma}\left(\left\|\boldsymbol{\theta}_{k-1, n_{\text {loop }}}\right\|_{\ell_{2}}\right)$, where $\left\|\boldsymbol{\theta}_{k-1, n_{\text {loop }}}\right\|_{\ell_{2}}$ is the disagreement vector with respect to $\mathcal{F}_{k-1}^{\star}$ at the end of the consensus stage during the $(k-1)^{\text {th }}$ time instant; and $\left\|\boldsymbol{\theta}_{k, 0}\right\|_{\ell_{2}}$ is the disagreement vector with respect to $\mathcal{F}_{k}^{\star}$ after the update stage during the $k^{\text {th }}$ time instant.

Next we obtain the minimum number of consensus loops for achieving $\varepsilon$-consensus across the network and also derive conditions on the communication network topology for a given number of consensus loops.

Theorem 7. (BCF-LogOP on SC Balanced Digraphs) Under Assumptions 5, 7, 8, and an acceptable communication error $\varepsilon_{\text {comm }}>0$, each agent tracks the target using the BCF algorithm. For some acceptable consensus error $\varepsilon_{\mathrm{cons}}>0$ and $\gamma_{k}=\min \left(\boldsymbol{\Gamma}\left(\left\|\boldsymbol{\theta}_{k-1, n_{\text {loop }}}\right\|_{\ell_{2}}\right), 2 \sqrt{m}\right)$ :

(i) for a given $P_{k}$, if the number of consensus loops $n_{\text {loop }}$ satisfies

$$
\left(\sigma_{m-1}\left(P_{k}\right)\right)^{n_{\text {loop }}} \gamma_{k}+2 n_{\text {loop }} \varepsilon_{\text {comm }} \sqrt{m} \leq \varepsilon_{\text {cons }} ;
$$

or (ii) for a given $n_{\mathrm{loop}}$, if the communication network topology $\left(P_{k}\right)$ during the $k^{\text {th }}$ time instant is such that 


$$
\sigma_{m-1}\left(P_{k}\right) \leq\left(\frac{\varepsilon_{\mathrm{cons}}-2 n_{\mathrm{loop}} \varepsilon_{\mathrm{comm}} \sqrt{m}}{\gamma_{k}}\right)^{\frac{1}{n_{\mathrm{loop}}}} ;
$$

then the $\ell_{2}$ norm of the disagreement vector at the end of the consensus stage is less than $\varepsilon_{\text {cons }}$, i.e., $\left\|\boldsymbol{\theta}_{k, n_{\text {loop }}}\right\|_{\ell_{2}} \leq \varepsilon_{\text {cons. }}$.

Proof: In the absence of communication inaccuracies, Theorem 5 states that the local estimated pdfs $\mathcal{F}_{k, 0}^{j}$ globally exponentially converges pointwise to a consensual pdf $\mathcal{F}_{k}^{\star}$ given by 14 with a rate of $\sigma_{m-1}\left(P_{k}\right)$, i.e. $\left\|\mathcal{F}_{k, \nu}^{j}-\mathcal{F}_{k}^{\star}\right\|_{\mathcal{L}_{1}} \leq$ $\left(\sigma_{m-1}\left(P_{k}\right)\right)^{\nu}\left\|\mathcal{F}_{k, 0}^{j}-\mathcal{F}_{k}^{\star}\right\|_{\mathcal{L}_{1}}$. If $\boldsymbol{\theta}_{k, 0}$ is the initial disagreement vector at the start of the consensus stage, then $\left\|\boldsymbol{\theta}_{k, n_{\text {loop }}}\right\|_{\ell_{2}} \leq$ $\left(\sigma_{m-1}\left(P_{k}\right)\right)^{n_{\text {loop }}}\left\|\boldsymbol{\theta}_{k, 0}\right\|_{\ell_{2}} \leq\left(\sigma_{m-1}\left(P_{k}\right)\right)^{n_{\text {loop }}} \gamma_{k}$.

In the presence of communication error, combining 26 with the previous result gives $\left\|\tilde{\mathcal{F}}_{k, \nu}^{j}-\mathcal{F}_{k}^{\star}\right\|_{\mathcal{L}_{1}} \leq$ $\left(\sigma_{m-1}\left(P_{k}\right)\right)^{\nu}\left\|\mathcal{F}_{k, 0}^{j}-\mathcal{F}_{k}^{\star}\right\|_{\mathcal{L}_{1}}+\nu \varepsilon_{\text {comm }}$. Since $\theta_{k, \nu}^{j} \leq \| \tilde{\mathcal{F}}_{k, \nu}^{j}-$ $\mathcal{F}_{k}^{\star} \|_{\mathcal{L}_{1}}+\nu \varepsilon_{\text {comm }}$, the disagreement vector after $n_{\text {loop }}$ iterations is given by $\left\|\boldsymbol{\theta}_{k, n_{\text {loop }}}\right\|_{\ell_{2}} \leq\left(\sigma_{m-1}\left(P_{k}\right)\right)^{n_{\text {loop }}}\left\|\boldsymbol{\theta}_{k, 0}\right\|_{\ell_{2}}+$ $2 n_{\text {loop }} \varepsilon_{\text {comm }} \sqrt{m}$. Thus, we get the conditions on $n_{\text {loop }}$ or $\sigma_{m-1}\left(P_{k}\right)$ from the inequality $\left(\sigma_{m-1}\left(P_{k}\right)\right)^{n_{\text {loop }}} \gamma_{k}+$ $2 n_{\text {loop }} \varepsilon_{\text {comm }} \sqrt{m} \leq \varepsilon_{\text {cons }}$.

Note that in the absence of communication inaccuracies, 27 simplifies to $n_{\text {loop }} \geq\left[\frac{\ln \left(\varepsilon_{\text {cons }} / \gamma_{k}\right)}{\ln \sigma_{m-1}\left(P_{k}\right)}\right]$ and 28 simplifies to $\sigma_{m-1}\left(P_{k}\right) \leq\left(\frac{\varepsilon_{\text {cons }}}{\gamma_{k}}\right)^{\frac{1}{n_{\text {loop }}}}$. In the particular case where $n_{\text {loop }}=1$ and communication errors are present, 28) simplifies to $\sigma_{m-1}\left(P_{k}\right) \leq \frac{\varepsilon_{\text {cons }}-2 \varepsilon_{\text {comm }} \sqrt{m}}{\gamma_{k}}$ and the necessary condition for a valid solution is $2 \varepsilon_{\text {comm }} \sqrt{m}<\varepsilon_{\text {cons }}$. In the genral case, it is desireable that $2 \varepsilon_{\text {comm }} \sqrt{m} \ll \varepsilon_{\text {cons }}$ for a valid solution to Theorem 7

\section{B. Hierarchical Bayesian Consensus Filtering}

In this section, we modify the original problem statement such that only $m_{1}$ out of $m$ agents are able to observe the target at the $k^{\text {th }}$ time instant. In this scenario, the other $m_{2}\left(=m-m_{1}\right)$ agents are not able to observe the target. Without loss of generality, we assume that the first $m_{1}$ agents, i.e., $\left\{1,2, \ldots, m_{1}\right\}$, are tracking the target. During the Bayesian filtering stage, each tracking agent (i.e., agent tracking the target) estimates the posterior pdf of the target's states at the $k^{\text {th }}$ time instant $\left(\mathcal{F}_{k}^{j}=p_{k}^{j}\left(\boldsymbol{x}_{k} \mid \boldsymbol{z}_{k}^{\mathcal{S}_{k}^{j} \cap\left\{1, \ldots m_{1}\right\}}\right), \forall j \in\right.$ $\left\{1, \ldots, m_{1}\right\}$ ) using the estimated prior pdf of the target's states $\left(\mathcal{F}_{k-1}^{j}\right)$ and the new measurement array $\boldsymbol{z}_{k}^{\mathcal{S}_{k}^{j} \cap\left\{1, \ldots m_{1}\right\}}:=$ $\left\{\boldsymbol{z}_{k}^{\ell}, \forall \ell \in \mathcal{S}_{k}^{j} \cap\left\{1, \ldots m_{1}\right\}\right\}$ obtained from the neighboring tracking agents. Each non-tracking agent (i.e., agent not tracking the target) only propagates its prior pdf during this stage to obtain $p_{k}^{j}\left(\boldsymbol{x}_{k}\right), \forall j \in\left\{m_{1}+1, \ldots, m\right\}$.

The objective of hierarchical consensus algorithm is to guarantee pointwise convergence of each $\mathcal{F}_{k, \nu}^{j}, \forall j \in\{1, \ldots, m\}$ to a pdf $\mathcal{F}_{k}^{\star}$ and only the local estimates of the agents tracking the target contribute to the consensual pdf. This is achieved by each tracking agent recursively transmitting its estimate of the target's states to other agents, only receiving estimates from its neighboring tracking agents and updating its estimate of the target. On the other hand, each non-tracking agent recursively transmits its estimate of the target's states to other agents, receives estimates from all its neighboring agents and updates its estimate of the target. This is illustrated using the pseudocode in Algorithm 2 and the following equations:

$$
\begin{aligned}
\mathcal{F}_{k, \nu}^{j} & =\mathcal{T}\left(\cup_{\ell \in \mathcal{J}_{k}^{j} \cap\left\{1, \ldots, m_{1}\right\}}\left\{\mathcal{F}_{k, \nu-1}^{\ell}\right\}\right), \\
\forall j & \in\left\{1, \ldots, m_{1}\right\}, \forall \nu \in \mathbb{N}, \\
\mathcal{F}_{k, \nu}^{j}= & \mathcal{T}\left(\cup_{\ell \in \mathcal{J}_{k}^{j}}\left\{\mathcal{F}_{k, \nu-1}^{\ell}\right\}\right), \\
\forall j & \in\left\{m_{1}+1, \ldots, m\right\}, \forall \nu \in \mathbb{N},
\end{aligned}
$$

where, $\mathcal{T}(\cdot)$ refers to the $\operatorname{LogOP}(8)$ for combining pdfs. Let $\mathcal{D}_{k}$ represent the communication network topology of only the tracking agents.

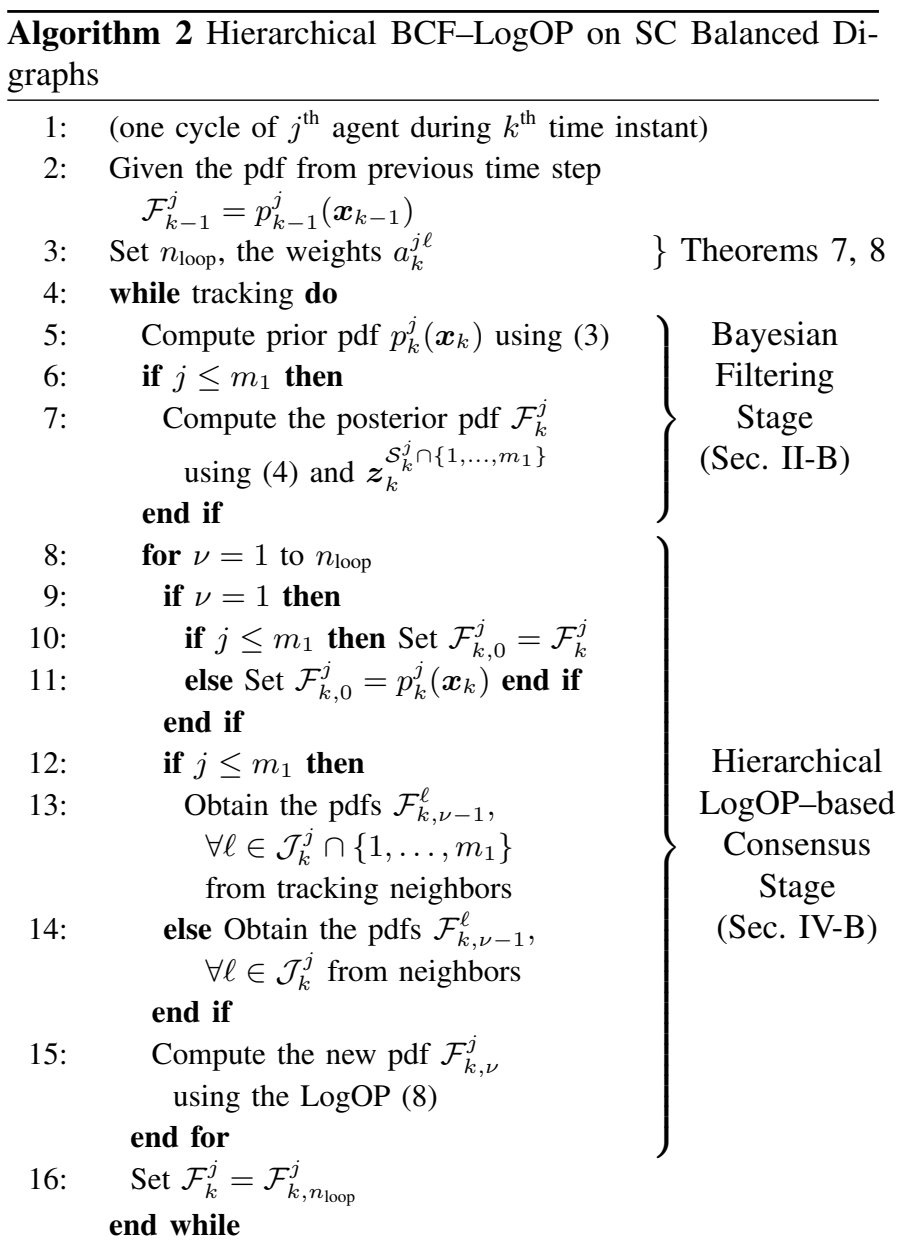

Assumption 9. The communication network topologies $\mathcal{G}_{k}$ and $\mathcal{D}_{k}$ are SC and the weights $a_{k}^{j \ell}$ are such that the digraph $\mathcal{D}_{k}$ is balanced. The weights $a_{k, \nu-1}^{j \ell}, \forall j, \ell \in\{1, \ldots, m\}$ and the matrix $P_{k, \nu-1}$ have the following properties: (i) The weights are the same for all consensus loops within each time instants, i.e., $a_{k, \nu-1}^{j \ell}=a_{k}^{j \ell}$ and $P_{k, \nu-1}=P_{k}, \forall \nu \in \mathbb{N}$. Moreover, $P_{k}$ can be decomposed into four parts $P_{k}=\left[\begin{array}{ll}P_{k 1} & P_{k 2} \\ P_{k 3} & P_{k 4}\end{array}\right]$, where $P_{k 1} \in \mathbb{R}^{m_{1} \times m_{1}}, P_{k 2}=\mathbb{R}^{m_{1} \times m_{2}}, P_{k 3} \in \mathbb{R}^{m_{2} \times m_{1}}$, and $P_{k 4} \in \mathbb{R}^{m_{2} \times m_{2}}$. (ii) If $j \in\left\{1, \ldots, m_{1}\right\}$, then $a_{k}^{j \ell}>0$ if and only if $\ell \in \mathcal{J}_{k}^{j} \cap\left\{1, \ldots, m_{1}\right\}$, else $a_{k}^{j \ell}=0$; hence $P_{k 2}=\mathbf{0}^{m_{1} \times m_{2}}$. Moreover, $P_{k 1}$ is balanced, i.e., $\sum_{\ell \in \mathcal{J}_{k}^{j}} a_{k}^{j \ell}=\sum_{r \mid j \in \mathcal{J}_{k}^{r}} a_{k}^{r j}$, where $j, \ell, r \in\left\{1, \ldots, m_{1}\right\}$; (iii) 
If $j \in\left\{m_{1}+1, \ldots, m\right\}$, then $a_{k}^{j \ell}>0$ if and only if $\ell \in \mathcal{J}_{k}^{j}$, else $a_{k}^{j \ell}=0$; (iv) The matrix $P_{k}$ is row stochastic, i.e., $\sum_{\ell=1}^{m} a_{k}^{j \ell}=1$.

Theorem 8. (Hierarchical Consensus using the LogOP on SC Balanced Digraphs) Under Assumptions 7 and 9. using the $\log O P$ (8), each $\mathcal{F}_{k, \nu}^{j}$ globally exponentially converges pointwise to the pdf $\mathcal{F}_{k}^{\star}$ given by:

$$
\mathcal{F}_{k}^{\star}=p_{k}^{\star}\left(\boldsymbol{x}_{k}\right)=\frac{\prod_{i=1}^{m_{1}}\left(p_{k, 0}^{i}\left(\boldsymbol{x}_{k}\right)\right)^{\frac{1}{m_{1}}}}{\int_{\mathcal{X}} \prod_{i=1}^{m_{1}}\left(p_{k, 0}^{i}\left(\boldsymbol{x}_{k}\right)\right)^{\frac{1}{m_{1}}} d \mu\left(\boldsymbol{x}_{k}\right)}
$$

at a rate faster or equal to $\sqrt{\lambda_{m_{1}-1}\left(P_{k 1}^{T} P_{k 1}\right)}=\sigma_{m_{1}-1}\left(P_{k 1}\right)$. Only the initial estimates of the tracking agents contribute to the consensual pdf $\mathcal{F}_{k}^{\star}$. Furthermore, their induced measures converge in total variation, i.e., $\lim _{\nu \rightarrow \infty} \mu_{\mathcal{F}_{k, \nu}^{j}} \stackrel{T . V .}{\longrightarrow} \mu_{\mathcal{F}_{k}^{\star}}, \forall j \in$ $\{1, \ldots, m\}$.

Proof: The matrix $P_{k 1}$ conforms to the balanced digraph $\mathcal{D}_{k}$. Let $\mathbf{1}_{m_{1}}=[1,1, \ldots, 1]^{T}$, with $m_{1}$ elements. Similar to the proof of Theorem 5, we get $P_{k 1}$ is a primitive matrix and $\lim _{\nu \rightarrow \infty} P_{k 1}^{\nu}=\frac{1}{m_{1}} \mathbf{1}_{m_{1}} \mathbf{1}_{m_{1}}^{T}$.

Next, we decompose $\mathcal{U}_{k, \nu}$ from equation 11 into two parts such that $\mathcal{U}_{k, \nu}=\left[\mathcal{Y}_{k, \nu} ; \mathcal{Z}_{k, \nu}\right]$, where $\mathcal{Y}_{k, \nu}=$ $\left(\mathcal{H}_{k, \nu}^{1}, \ldots, \mathcal{H}_{k, \nu}^{m_{1}}\right)^{T}$ and $\mathcal{Z}_{k, \nu}=\left(\mathcal{H}_{k, \nu}^{m_{1}+1}, \ldots, \mathcal{H}_{k, \nu}^{m}\right)^{T}$. Since $P_{k 2}$ is a zero matrix, (11) generalizes and hierarchically decomposes to:

$$
\begin{aligned}
& \mathcal{Y}_{k, \nu+1}=P_{k 1}^{\nu} \mathcal{Y}_{k, 0}, \forall \nu \in \mathbb{N} \\
& \mathcal{Z}_{k, \nu+1}=P_{k 3} \mathcal{Y}_{k, \nu}+P_{k 4} \mathcal{Z}_{k, \nu}, \forall \nu \in \mathbb{N}
\end{aligned}
$$

Combining equation (32) with the previous result gives $\lim _{\nu \rightarrow \infty} \mathcal{Y}_{k, \nu}=\frac{1}{m_{1}} \mathbf{1}_{m_{1}} \mathbf{1}_{m_{1}}^{T} \mathcal{Y}_{k, 0}$. Thus $\lim _{\nu \rightarrow \infty} \mathcal{H}_{k, \nu}^{j}=$ $\mathcal{H}_{k}^{\star}=\frac{1}{m_{1}} \mathbf{1}_{m_{1}}^{T} \mathcal{Y}_{k, 0}=\frac{1}{m_{1}} \sum_{i=1}^{m_{1}} \mathcal{H}_{k, 0}^{i}, \forall j \in\left\{1, \ldots, m_{1}\right\}$. From the proof of Theorem 5 , we get $\mathcal{F}_{k, \nu}^{j}, \forall j \in\left\{1, \ldots, m_{1}\right\}$ globally exponentially converges pointwise to $\mathcal{F}_{k}^{\star}$ given by (31) with a rate faster or equal to $\sigma_{m_{1}-1}\left(P_{k 1}\right)$.

Since $\mathcal{G}(k)$ is strongly connected, information from the tracking agents reach the non-tracking agents. Taking the limit of equation (33) and substituting the above result gives:

$$
\lim _{\nu \rightarrow \infty} \mathcal{Z}_{k, \nu+1}=\frac{1}{m_{1}} P_{k 3} \mathbf{1}_{m_{1}} \mathbf{1}_{m_{1}}^{T} \mathcal{Y}_{k, 0}+P_{k 4} \lim _{\nu \rightarrow \infty} \mathcal{Z}_{k, \nu}
$$

Let $\mathbf{1}_{m_{2}}=[1,1, \ldots, 1]^{T}$, with $m_{2}$ elements. Since $P_{k}$ is row stochastic, we get $P_{k 3} \mathbf{1}_{m_{1}}=\left[\mathbf{I}-P_{k 4}\right] \mathbf{1}_{m_{2}}$. Hence, from equation 34 , we get $\lim _{\nu \rightarrow \infty} \mathcal{Z}_{k, \nu}=\frac{1}{m_{1}} \mathbf{1}_{m_{2}} \mathbf{1}_{m_{1}}^{T} \mathcal{Y}_{k, 0}$. Moreover, the inessential states die out geometrically fast [68. pp. 120]. Hence $\lim _{\nu \rightarrow \infty} \mathcal{H}_{k, \nu}^{j}=\mathcal{H}_{k}^{\star}=\frac{1}{m_{1}} \mathbf{1}_{m_{1}}^{T} \mathcal{Y}_{k, 0}, \forall j \in$ $\left\{m_{1}+1, \ldots, m\right\}$. Hence, the estimates of the non-tracking agents $\mathcal{F}_{k, \nu}^{j}, \forall j \in\left\{m_{1}+1, \ldots, m\right\}$ also converge pointwise geometrically fast to the same consensual pdf $\mathcal{F}_{k}^{\star}$ given by 31 . By Lemma 2 we get $\lim _{\nu \rightarrow \infty} \mu_{\mathcal{F}_{k, \nu}^{j}} \stackrel{\text { T.V. }}{\longrightarrow} \mu_{\mathcal{F}_{k}^{*}}, \forall j \in$
$\{1, \ldots, m\}$.

Note that Theorem 7 can be directly applied from Section IV-A to find the minimum number of consensus loops $n_{\text {loop }}$ for achieving $\epsilon$-convergence in a given communication network topology or for designing the $P_{k 1}$ matrix for a given number of consensus loops. A simulation example of Hierarchical

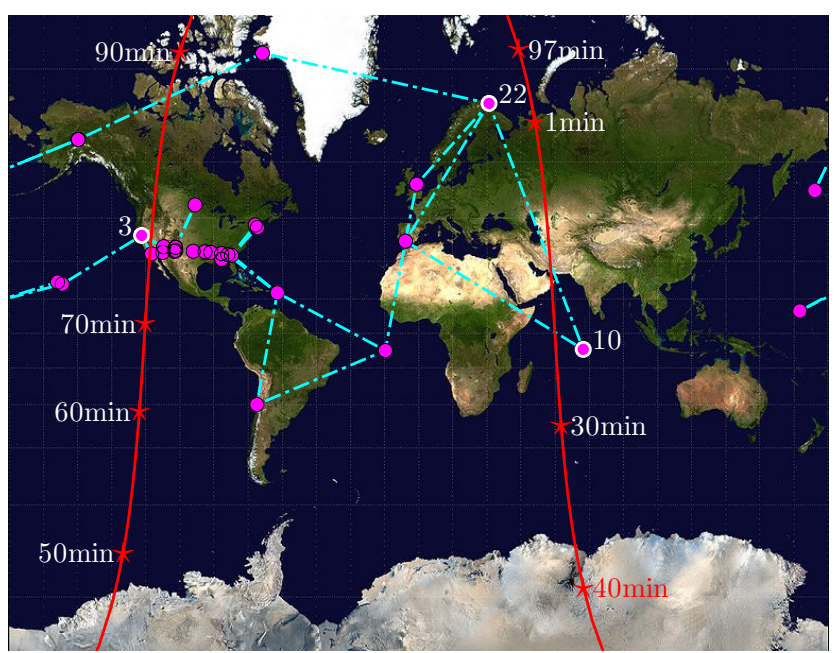

Fig. 5. The SSN locations are shown along with their static SC balanced communication network topology. The orbit of the Iridium-33 debris is shown in red, where $\star$ marks its actual position during particular time instants.

$\mathrm{BCF}-\mathrm{LogOP}$ algorithm for tracking orbital debris in space is discussed in the next section.

\section{NumERicAl EXAMPLE}

Currently, there are over ten thousand objects in Earth orbit, of size $0.5 \mathrm{~cm}$ or greater, and almost $95 \%$ of them are nonfunctional space debris. These debris pose a significant threat to functional spacecraft and satellites in orbit. The US has established the Space Surveillance Network (SSN) for ground based observations of the orbital debris using radars and optical telescopes [69], [70]. In February 2009, the Iridium-33 satellite collided with the Kosmos-2251 satellite and a large number of debris fragments were created. In this section, we use the Hierarchical BCF-LogOP Algorithm to track one of the Iridium-33 debris created in this collision. The orbit of this debris around Earth and the location of SSN sensors are shown in Fig. 5

The actual two-line element set (TLE) of the Iridium33 debris was accessed from North American Aerospace Defense Command (NORAD) on $4^{\text {th }}$ Dec 2013. The nonlinear Simplified General Perturbations (SGP4) model, which uses an extensive gravitational model and accounts for the drag effect on mean motion [71], [72], is used as the target dynamics model. The communication network topology of the SSN is assumed to be a static SC balanced graph, as shown in Figure 5 If the debris is visible above the sensor's horizon, then it is assumed to create a single measurement during each time step of one minute. The heterogeneous measurement model of the $j^{\text {th }}$ sensor is given by:

$$
\boldsymbol{z}_{k}^{j}=\boldsymbol{x}_{k}+\boldsymbol{w}_{k}^{j}, \text { where } \boldsymbol{w}_{k}^{j}=\mathcal{N}(0,(1000+50 j) \times \mathbf{I}),
$$

where $\boldsymbol{x}_{k} \in \mathbb{R}^{3}$ is the actual location of the debris and the additive Gaussian measurement noise depends on the sensor number. Since it is not possible to implement the SGP4 target dynamics on distributed estimation algorithms discussed in the literature [11]-[20], we compare the performance of our Hierarchical BCF-LogOP algorithm (Algorithm 2) against the 


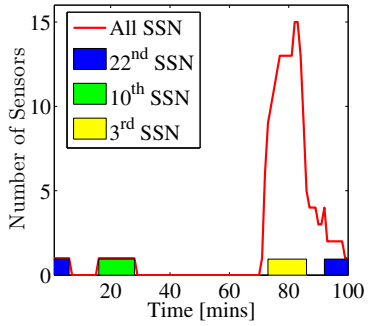

(a)

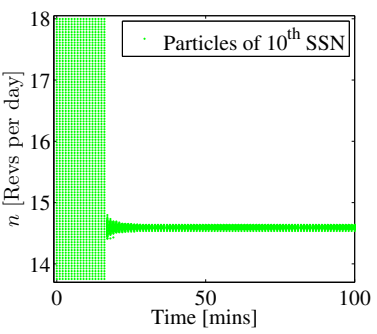

(c)

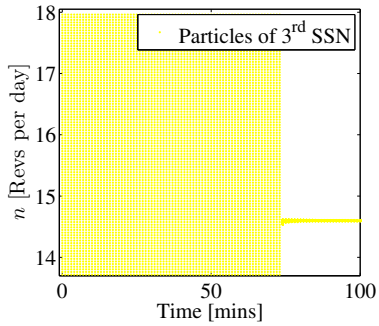

(b)

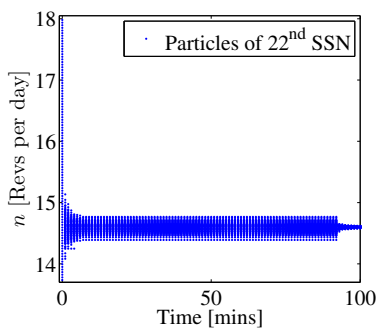

(d)
Fig. 6. (a) Number of SSN sensors observing debris. Trajectories of particles for stand-alone Bayesian filters for (b) $3^{\text {rd }}$, (c) $10^{\text {th }}$, and (d) $22^{\text {nd }}$ SSN sensor.

Hierarchical BCF-LinOP algorithm, where the LinOP is used during the consensus stage.

In this simulation example, we simplify the debris tracking problem by assuming only the mean motion $(n)$ of the debris is unknown. The objective of this simulation example is to estimate $n$ of the Iridium-33 debris within 100 minutes. Hence, each sensor knows the other TLE parameters of the debris and an uniform prior distribution $\left(\mathcal{F}_{0}^{j}\right)$ is assumed. Note that at any time instant, only a few of the SSN sensors can observe the debris, as shown in Fig 6(a). The results of three stand-alone Bayesian filters, implemented using particle filters with resampling [36], are shown in Fig 6(b-d). Note that the estimates of the $22^{\text {nd }}$ and $10^{\text {th }}$ sensors initially do not converge due to large measurement error, in spite of observing the debris for some time. The estimates of the $3^{\text {rd }}$ sensor does converge when it is able to observe the debris after 70 minutes. Hence we propose to use the Hierarchical BCF-LogOP algorithm where the consensual distribution is updated as and when sensors observe the debris.

Particle filters with resampling are used to evaluate the Bayesian filters and communicate pdfs in the Hierarchical BCF algorithms. 100 particles are used by each sensor and 10 consensus loops are executed during each time step of one minute. The trajectories of all the particles of the sensors in the Hierarchical BCF algorithm using LinOP and LogOP and their respective consensual probability distributions at different time instants are shown in Figure 6(a-d). As expected, all the sensors converge on the correct value of $n$ of 14.6 revs per day. The Hierarchical BCF-LinOP estimates are multimodal for the first 90 minutes. On the other hand, the Hierarchical BCF-LogOP estimates converges to the correct value within the first 10 minutes because the LogOP algorithm efficiently communicates the best consensual estimate to other sensors during each time step and achieves consensus across the network.

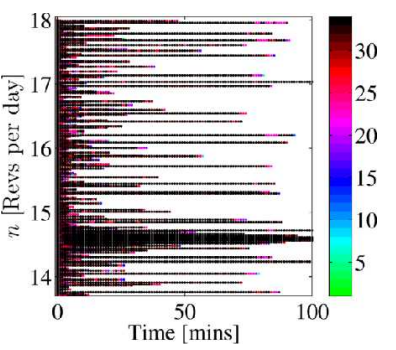

(a)

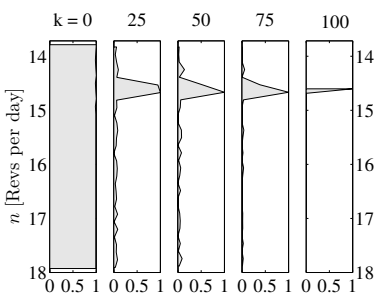

(c)

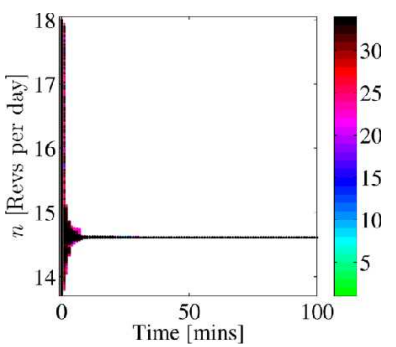

(b)

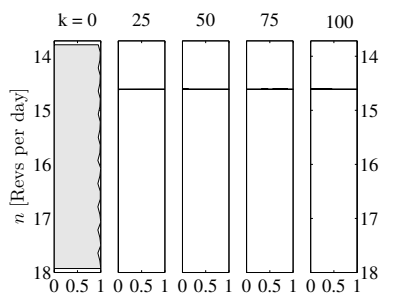

(d)
Fig. 7. Trajectories of particles of all sensors for (a) Hierarchical BCFLinOP and (b) Hierarchical BCF-LogOP. The color-bar on the right denotes the 33 SSN sensors. Evolution of the consensual probability distribution for (c) Hierarchical BCF-LinOP and (d) Hierarchical BCF-LogOP.

\section{CONCLUSION}

In this paper, we extended the scope of distributed estimation algorithms in a Bayesian filtering framework in order to simultaneously track targets, with general nonlinear timevarying target dynamic models, using a strongly connected network of heterogeneous agents, with general nonlinear timevarying measurement models. We introduced the Bayesian filter with/without measurement exchange to generate local estimated pdfs of the target's states. We compared the LinOP and LogOP methods of combining local posterior pdfs and determined that $\operatorname{LogOP}$ is the superior scheme. The LogOP algorithm on SC balanced digraph converges globally exponentially, and the consensual pdf minimizes the information lost during the consensus stage because it minimizes the sum of KL divergences to each locally estimated probability distribution. We also explored several methods of communicating pdfs across the sensor network. We introduced the BCF algorithm, where the local estimated posterior pdfs of the target's states are first updated using the Bayesian filter and then recursively combined during the consensus stage using $\log \mathrm{OP}$, so that the agents can track a moving target and also maintain consensus across the network. Conditions for exponential convergence of the $\mathrm{BCF}$ algorithm and constraints on the communication network topology have been studied. The Hierarchical BCF algorithm, where some of the agents do not observe the target, has also been investigated. Simulation results demonstrate the effectiveness of the BCF algorithms for nonlinear distributed estimation problems.

\section{ACKNOWLEDGMENT}

The authors would like to thank F. Hadaegh, D. Bayard, S. Hutchinson, P. Voulgaris, M. Egerstedt, A. Gupta, A. Dani, D. Morgan, S. Sengupta, and A. Olshevsky for stimulating discussions about this paper. 


\section{APPENDIX A}

\section{PROOF OF THEOREM 3}

Under Assumption 6, $P_{k}$ is a nonnegative, row stochastic and irreducible matrix. Similar to the proof in [6], all the diagonal entries of $P_{k}$ are positive, then $P_{k}^{m-1}>0$ and [73, Theorem 8.5.2, pp. 516] implies that $P_{k}$ is a primitive matrix. Since $P_{k}$ is a regular matrix, it has only one recurrent class which is aperiodic [68, pp. 127]. Since $P_{k}$ is row stochastic, 1 is its right eigenvector corresponding to the eigenvalue 1 , i.e., $P_{k} \mathbf{1}=11$. Moreover, according to the Gershgorin Disc Theorem (cf. [73, pp. 344]), all the eigenvalues of $P_{k}$ are located in the unit circle, i.e. the spectral radius $\rho\left(P_{k}\right)=1$. Hence Perron-Frobenius theorem (cf. [68, pp. 3]) states that there exists a left eigenvector $\pi$ of $P_{k}$ corresponding to the eigenvalue 1 which is unique to constant multiples, i.e., $P_{k}^{T} \boldsymbol{\pi}=1 \boldsymbol{\pi}$. The ergodic theorem for primitive Markov chains (cf. [68, pp. 119]) states that $P_{k}$ has an unique stationary distribution given by the solution of the normalizing condition $\boldsymbol{\pi}^{T} \mathbf{1}=1$ and $\lim _{\nu \rightarrow \infty} P_{k}^{\nu}=\mathbf{1} \boldsymbol{\pi}^{T}$.

Obviously, (7) generalizes to $\mathcal{W}_{k, \nu}=P_{k}^{\nu} \mathcal{W}_{k, 0}, \forall \nu \in$ $\mathbb{N}$, where $\mathcal{W}_{k, 0}=\left(\mathcal{F}_{k, 0}^{1}, \ldots, \mathcal{F}_{k, 0}^{m}\right)^{T}$. Hence we get $\lim _{\nu \rightarrow \infty} \mathcal{W}_{k, \nu}=\mathbf{1} \boldsymbol{\pi}^{T} \mathcal{W}_{k, 0}$. Thus, each $\mathcal{F}_{k, \nu}^{j}$ converges pointwise to the consensual pdf $\mathcal{F}_{k}^{\star}=\pi^{T} \mathcal{W}_{k, 0}$. Note that $\sum_{i=1}^{m} \pi_{i}=1$, as needed for $\mathcal{F}_{k}^{\star}$ to be a valid probability distribution, where $\pi_{i}$ is the individual element of the vector $\pi$. By Lemma 2, the measure induced by $\mathcal{F}_{k, \nu}^{j}$ on $\mathscr{X}$ converges in total variation to the measure induced by $\mathcal{F}_{k}^{\star}$ on $\mathscr{X}$, i.e., $\lim _{\nu \rightarrow \infty} \mu_{\mathcal{F}_{k, \nu}^{j}} \stackrel{\text { T.V. }}{\longrightarrow} \mu_{\mathcal{F}_{k}^{\star}}$.

\section{REFERENCES}

[1] R. Olfati-Saber and R. Murray, "Consensus problems in networks of agents with switching topology and time-delays," IEEE Trans. Autom. Control, vol. 49, no. 9, pp. 1520 - 1533, 2004.

[2] A. Jadbabaie, J. Lin, and A. S. Morse, "Coordination of groups of mobile autonomous agents using nearest neighbor rules," IEEE Trans. Autom. Control, vol. 48, no. 6, pp. 988 - 1001, 2003.

[3] S. Boyd, A. Ghosh, B. Prabhakar, and D. Shah, "Randomized gossip algorithms," IEEE Trans. Inf. Theory, vol. 52, pp. 2508 - 2530, June 2006.

[4] L. Xiao and S. Boyd, "Fast linear iterations for distributed averaging," Syst. Control Lett., vol. 53, pp. 65 - 78, 2004

[5] W. Ren and R. W. Beard, "Consensus seeking in multiagent systems under dynamically changing interaction topologies," IEEE Trans. Autom. Control, vol. 50, pp. 655 - 661, May 2005.

[6] R. Olfati-Saber, J. A. Fax, and R. M. Murray, "Consensus and cooperation in networked multi-agent systems," Proc. of the IEEE, vol. 95, no. 1 , pp. 215-233, 2007.

[7] V. D. Blondel, J. M. Hendrickx, A. Olshevsky, and J. N. Tsitsiklis, "Convergence in multiagent coordination, consensus, and flocking," in 44th IEEE Conf. Decision Control, (Seville, Spain), Dec. 2005.

[8] J. Tsitsiklis, D. Bertsekas, and M. Athans, "Distributed asynchronous deterministic and stochastic gradient optimization algorithms," IEEE Trans. Autom. Control, vol. 31, no. 9, pp. 803 - 812, 1986.

[9] M. Zhu and S. Martínez, "On distributed optimization under inequality and equality constraints via penalty primal-dual methods," in Amer. Control Conf., (Baltimore, US), pp. 2434-2439, 2010.

[10] A. Nedic and A. Ozdaglar, Convex Optimization in Signal Processing and Communications, ch. Cooperative distributed multi-agent optimization, pp. 340 - 386. Cambridge University Press, 2009.

[11] V. Borkar and P. Varaiya, "Asymptotic agreement in distributed estimation," IEEE Trans. Autom. Control, vol. 27, no. 3, pp. 650 - 655, 1982.

[12] S. Kar and J. M. F. Moura, "Convergence rate analysis of distributed gossip (linear parameter) estimation: Fundamental limits and tradeoffs," IEEE J. Sel. Topics Signal Process., vol. 5, pp. 674 - 690, Aug 2011.
[13] I. Schizas, G. Mateos, and G. Giannakis, "Distributed LMS for consensus-based in-network adaptive processing," IEEE Trans. Signal Process., vol. 57, pp. 2365 - 2382, June 2009.

[14] D. Spanos, R. Olfati-Saber, and R. M. Murray, "Distributed sensor fusion using dynamic consensus," in Proc. IFAC, 2005.

[15] M. Coates, "Distributed particle filters for sensor networks," in Proc. 3rd Int. Symp. Inform. Process. Sensor Networks, (New York, USA), pp. 99-107, 2004.

[16] F. Zhang and N. E. Leonard, "Cooperative filters and control for cooperative exploration," IEEE Trans. Autom. Control, vol. 55, no. 3, pp. $650-663,2010$.

[17] N. Ahmed, J. Schoenberg, and M. Campbell, Robotics: Science and Systems VIII, ch. Fast Weighted Exponential Product Rules for Robust General Multi-Robot Data Fusion, pp. 9-16. MIT Press, 2013.

[18] M. Demetriou and D. Uciński, "State estimation of spatially distributed processes using mobile sensing agents," in Amer. Control Conf., (San Francisco, CA, USA), pp. 1770-1776, 2011.

[19] A. Dimakis, S. Kar, J. Moura, M. Rabbat, and A. Scaglione, "Gossip algorithms for distributed signal processing," Proc. of the IEEE, vol. 98, no. 11, pp. 1847-1864, 2010.

[20] B. Açikmeşe, F. Y. Hadaegh, D. P. Scharf, and S. R. Ploen, "Formulation and analysis of stability for spacecraft formations," IET Control Theory Appl., vol. 1, no. 2, pp. 461-474, 2007.

[21] Y. Xu, V. Gupta, and C. Fischione, "Distributed estimation," in Ereference Signal Processing, Elsevier, 2013. Editors: Rama Chellappa, Sergios Theodoridis.

[22] R. Olfati-Saber, "Kalman-consensus filter : Optimality, stability, and performance," in 48th IEEE Conf. Decision Control, (Shanghai, China), pp. 7036-7042, December 2009.

[23] M. H. DeGroot, Probability and Statistics. Cambridge, Massachusetts: Addison-Wesley, 1975.

[24] H. Jeffreys, Theory of Probability. Oxford: Clarendon Press, 1961.

[25] K. Subrahmaniam, A Primer in Probability. New York, NY: M. Dekker, 1979.

[26] J. Pearl, Probabilistic Reasoning in Intelligent Systems: Networks of Plausible Inference. San Mateo, CA: Morgan Kaufmann, 1988.

[27] R. E. Kalman, "A new approach to linear filtering and prediction problems," Trans. ASME J. Basic Eng., vol. 82, no. Series D, pp. 35-45, 1960.

[28] S. J. Julier and J. K. Uhlmann, "Unscented filtering and nonlinear estimation," Proc. of the IEEE, vol. 92, no. 3, pp. 401-422, 2004.

[29] S. Thrun, "Probabilistic algorithms in robotics," AI Magazine, vol. 21, no. 4, pp. 93-109, 2000.

[30] W. Burgard, D. Fox, D. Hennig, and T. Schmidt, "Estimating the absolute position of a mobile robot using position probability grids," in Proc. of the 14th Nat. Conf. Artificial Intell., August 1996.

[31] J. Diard, P. Bessière, and E. Mazer, "A survey of probabilistic models, using the Bayesian programming methodology as a unifying framework," in 2nd Int. Conf. Computational Intell., Robotics and Autonomous Syst., (Singapore), Dec. 2003.

[32] S. Thrun, W. Burgard, and D. Fox, Probabilistic Robotics. Cambridge, Massachusetts: The MIT Press, 2005.

[33] C. Boutilier, T. Dean, and S. Hanks, "Decision-theoretic planning: Structural assumptions and computational leverage," J. Artificial Intell. Research, vol. 11, pp. 1-94, 1999.

[34] L. P. Kaelbling, M. L. Littman, and A. R. Cassandra, "Planning and acting in partially observable stochastic domains," Artificial Intell., vol. 101, pp. 99-134, May 1998.

[35] O. Punska, "Bayesian approaches to multi-sensor data fusion," Master's thesis, Dept. of Eng., Univ. of Cambridge, 1999.

[36] M. S. Arulampalam, S. Maskell, N. Gordon, and T. Clapp, "A tutorial on particle filters for online nonlinear/non-Gaussian Bayesian tracking," IEEE Trans. Signal Process., vol. 50, pp. 174-188, February 2002.

[37] O. Lebeltel, P. Bessiere, J. Diard, and E. Mazer, "Bayesian robot programming," Autonomous Robots, vol. 16, pp. 49-79, January 2004.

[38] M.-H. Chen, "Bayesian computation: From posterior densities to Bayes factors, marginal likelihoods, and posterior model probabilities," in Bayesian Thinking, Modeling and Computation (D. K. Dey and C. R. Rao, eds.), Handbook of Statistics, ch. 15, pp. 437 - 457, Amsterdam: Elsevier, 2005.

[39] M. H. DeGroot, "Reaching a consensus," J. Amer. Statistical Assoc., vol. 69, no. 345, pp. $688-704,1960$.

[40] C. Genest and J. V. Zidek, "Combining probability distributions: A critique and an annotated bibliography," Statistical Sci., vol. 1, no. 1, pp. $114-135,1986$.

[41] M. Bacharach, "Normal Bayesian dialogues," J. Amer. Statistical Assoc., vol. 74 , no. 368 , pp. $837-846,1979$. 
[42] S. Chatterjee and E. Seneta, "Towards consensus: Some convergence theorems on repeated averaging," J. Appl. Probability, vol. 14, no. 1, pp. $89-97,1977$.

[43] S. French, "Consensus of opinion," European J. Operational Research, vol. 7, pp. $332-340,1981$.

[44] P. Velagapudi, O. Prokopyev, K. Sycara, and P. Scerri, "Maintaining shared belief in a large multiagent team," in Proc. of FUSION, 2007.

[45] S. Yüksel, "Stochastic nestedness and the belief sharing information pattern," IEEE Trans. Autom. Control, vol. 54, no. 12, pp. 2773-2786, 2009.

[46] C. S. R. Fraser, L. F. Bertuccelli, H.-L. Choi, and J. P. How, "A hyperparameter consensus method for agreement under uncertainty," Automatica, vol. 48, no. 2, pp. 374 - 380, 2012.

[47] O. Hlinka, O. Slučiak, F. Hlawatsch, P. M. Djuric, and M. Rupp, "Likelihood consensus and its application to distributed particle filtering," IEEE Trans. Signal Process., vol. 60, no. 8, pp. 4334-4349, 2012.

[48] G. Pavlin, P. Oude, M. Maris, J. Nunnink, and T. Hood, "A multi-agent systems approach to distributed Bayesian information fusion," Inform. Fusion, vol. 11, pp. 267-282, 2010.

[49] A. Jadbabaie, P. Molavi, A. Sandroni, and A. Tahbaz-Salehi, "NonBayesian social learning," Games and Economic Behavior, vol. 76, pp. 210-225, 2012.

[50] K. A. Ross, Elementary Analysis: The Theory of Calculus. Springer, 1980.

[51] F. Daum, "Nonlinear filters: beyond the Kalman filter," in IEEE Aerospace Electron. Syst. Mag., vol. 20, pp. 57-69, 2005.

[52] T. M. Cover and J. A. Thomas, Elements of Information Theory. New York, NY: Wiley, 1991.

[53] T. Zhao and A. Nehorai, "Distributed sequential Bayesian estimation of a diffusive source in wireless sensor networks," IEEE Trans. Signal Process., vol. 55, no. 4, pp. 1511 - 1524, 2007.

[54] R. Durrett, Probability: Theory and Examples. Thomson Brooks, 2005.

[55] B. J. Julian, M. Angermann, M. Schwager, and D. Rus, "Distributed robotic sensor networks: An information-theoretic approach," Inter. J. of Robotics Research, vol. 31, no. 10, pp. 1134-1154, 2012.

[56] S. Weerahandi and J. V. Zidek, "Elements of multi-Bayesian decision theory," Ann. of Stat., vol. 11, no. 4, pp. 1032 - 1046, 1983.

[57] J. F. Nash, Jr, "The bargaining problem," Econometrica, vol. 18, no. 2, pp. $155-162,1950$.

[58] M. J. Rufo, J. Martín, and C. J. Pérez, "Log-linear pool to combine prior distributions: A suggestion for a calibration-based approach," Bayesian Analysis, vol. 7, no. 2, pp. 411-438, 2012.

[59] A. Smith, T. Cohn, and M. Osborne, "Logarithmic opinion pools for conditional random fields," in Proc. Assoc. Computational Linguistics, (Ann Arbor, Michigan), pp. 18-25, 2005.

[60] G. L. Gilardoni and M. K. Clayton, "On reaching a consensus using DeGroot's iterative pooling," Ann. Stat., vol. 21, no. 1, pp. 391 - 401, 1993.

[61] S.-J. Chung, S. Bandyopadhyay, I. Chang, and F. Y. Hadaegh, "Phase synchronization control of complex networks of Lagrangian systems on adaptive digraphs," Automatica, vol. 49, pp. 1148-1161, May 2013.

[62] B. D. O. Anderson and J. B. Moore, Optimal Filtering. Mineola, New York: Dover Publications, 2005.

[63] D. A. Reynolds, "Gaussian mixture models," Encyclopedia of Biometric Recognition, February 2008.

[64] G. J. McLachlan and K. E. Basford, Mixture models : inference and applications to clustering. New York, N.Y.: M. Dekker, 1988.

[65] J. H. Kotecha and P. M. Djuric, "Gaussian sum particle filtering," IEEE Trans. Signal Process., vol. 51, pp. 2602-2612, Oct. 2003.

[66] G. Kramer and S. A. Savari, "Communicating probability distributions," IEEE Trans. Inf. Theory, vol. 53, pp. 518-525, February 2007.

[67] S.-Y. Tu and A. H. Sayed, "Diffusion strategies outperform consensus strategies for distributed estimation over adaptive networks," IEEE Trans. Signal Process., vol. 60, pp. 6217-6234, Dec. 2012.

[68] E. Seneta, Non-negative Matrices and Markov Chains. New York, NY: Springer-Verlag, 2006.

[69] M. E. P. Chatters and M. B. J. Crothers, AU-18 Space Primer, ch. Space Surveillance Network, pp. 249-258. Air University Press, Maxwell Air Force Base, Alabama, 2009.

[70] D. A. Vallado and J. D. Griesbach, "Simulating space surveillance networks," in AAS/AIAA Astrodynamics Specialist Conf., (Girdwood), 2012. Paper AAS 11-580.

[71] F. R. Hoots and R. L. Roehrich, "Spacetrack report number 3: Models for propagation of NORAD element sets," tech. rep., U.S. Air Force Aerospace Defense Command, Colorado Springs, CO., 1980.

[72] D. Vallado and P. Crawford, "SGP4 orbit determination," in AIAA/AAS Astrodynamics Specialist Conf., 2008.
[73] R. A. Horn and C. R. Johnson, Matrix Analysis. Cambridge, England: Cambridge University Press, 1985. 